\title{
Prognostic Significance and Therapeutic Target of CXC Chemokines in the Microenvironment of Lung Adenocarcinoma
}

\author{
Kun Wang, Rongyang Li, Yu Zhang, Weifeng Qi, Tao Fang, Weiming Yue, Hui Tian \\ Department of Thoracic Surgery, Qilu Hospital, Cheeloo College of Medicine, Shandong University, Jinan, Shandong, 2500I2, People's Republic of \\ China \\ Correspondence: Hui Tian, Department of Thoracic Surgery, Qilu Hospital, Cheeloo College of Medicine, Shandong University, Jinan, Shandong, \\ 2500 I2, People's Republic of China, Email tianhuiql@email.sdu.edu.cn
}

\begin{abstract}
Background: Lung adenocarcinoma (LUAD) is one of the most important subtypes of lung cancer and has a high morbidity and mortality. Inflammatory CXC chemokines in tumor microenvironment can stimulate tumor growth, invasion, and metastasis, affecting the prognosis of patients. However, the differential expression profiles, prognostic values, and specific mechanisms of the CXC chemokine family in LUAD have not been clarified.

Methods: Transcriptome expression profile data were extracted from TIMER and TCGA. GEPIA was used to compare the relationship between CXC chemokines and clinicopathologic parameters. The prognostic analysis was performed using a Kaplan-Meier curve in GEPIA. LinkedOmics and TRRUST were applied to conduct the enrichment analysis of the regulatory networks containing the kinase targets, miRNA targets, and transcriptional factor targets. The characteristics of immune infiltration and immune-related clinical outcomes were evaluated with TIMER algorithms. Single-cell RNA sequencing localization analysis of genes as prognostic biomarkers were performed by PanglaoDB.

Results: Nine differentially expressed genes were identified in LUAD compared to normal tissues. Aberrant expression of CXCL2 $(P=0.0017), \operatorname{CXCL13}(P=0.0271), \operatorname{CXCL16}(P=0.016)$, and CXCL17 $(P=2.14 \mathrm{e}-5)$ was significantly correlated with clinical cancer stage. Furthermore, patients with low gene transcription of CXCL $7(P=0.017)$ and high expression of CXCL $17(P=0.00045)$ had a better prognosis in LUAD. We also found that immune cell infiltration was significantly correlated with LUAD microenvironment mediated by CXC chemokines. Cox proportional hazard model test was conducted and indicated that B cell infiltration could prolong the survival of the LUAD patients. CXCL17 exerted anti-tumors effect through pulmonary alveolar type II cells according to singlecell analysis.

Conclusion: Our research identified the aberrant expression profiles and prognostic biomarkers of CXC chemokines in LUAD. This detailed analysis of the regulatory factor networks for CXC chemokine gene expression may provide novel insights for selecting potential immunotherapeutic targets.
\end{abstract}

Keywords: CXC chemokines, lung adenocarcinoma, immune targets, biomarkers

\section{Introduction}

According to the latest statistics, lung cancer has become the leading cause of cancer morbidity and death worldwide. ${ }^{1}$ As the most common histological type, it accounts about $40 \%$ of all lung cancers. ${ }^{2}$ The comprehensive multimode treatment of lung cancer based on Thoracic Surgery has advanced considerably, providing improvement to patient prognosis. Therapy based on targeting immune checkpoints, especially anti-CTLA-4 and anti-PD-1/PD-L1 monoclonal antibody monotherapies, has also made significant progress in treating advanced lung cancer. ${ }^{3}$ However, given certain biological conditions, including low tumor mutation load, absence of antigen presentation, and the expression of inhibitory signals includingPD-L1, the effective clinical rate of immunotherapy for lung cancer is still only $20-30 \%$. Furthermore, the CEA, CA125, and CYFRA21-1 biomarkers are widely used in the clinical diagnosis and prognostic prediction of lung 
cancer, and they do not show sufficient sensitivity or specificity. Therefore, it is important to find specific, sensitive, and efficient tumor markers in the early diagnosis, therapeutic evaluation, and prognosis prediction of LUAD.

Chemokines, a family of approximately 50 low-molecular-weight molecules, are usually divided into 2 functional groups: inflammatory chemokines and homeostatic chemokines. Harmful stimulation usually induces inflammatory chemokines, including infection or oxidative damage, while homeostatic chemokines are structurally expressed in the regulation of normal immune networks. Chemotactic cytokines play an important role in many biological processes, including leukocyte recruitment and angiogenesis. ${ }^{4,5}$ Recent research has shown that chemokines can directly or indirectly regulate the tumor immunity microenvironment (TIM) and biological phenotype, affecting angiogenesis, tumorigenesis, metastasis of malignancy, therapeutic effect, and prognosis in patients. ${ }^{6}$ The systematic research on CXC chemokines would further reveal the mechanism of tumorigenesis or immune escape in the MIT, and contribute to finding suitable immune targets in this procedure. Some studies have demonstrated that the binding ofCXCL8tothe CXCR1 receptor induces lung cancer cell proliferation and promotes tumor cell metastasis, and overexpression of CXCL8 has been correlated with the poor prognosis of non-small cell lung cancer (NSCLC) patients. ${ }^{7}$ Moreover, CXCL4 and CXCL10 have been confirmed to attract antineoplastic dendritic cells, T lymphocytes, and NK cells, activating their antineoplastic immune response to inhibit tumor occurrence and development. ${ }^{8}$

Previous studies in this area were mainly unilateral studies on a single CXC chemokine mechanism and lacked systematic differential expression analysis and clinical prognosis prediction. Elucidation of the transcriptome biological process of the CXC chemokine family could comprehensively expand the understanding of the chemokine biological network and clarify its mechanism of tumorigenesis. The rapid development of second-generation gene sequencing technology and the establishment of various databases has contributed to analyzing CXC chemokines family comprehensively. ${ }^{9}$ Therefore, using several large public databases, we conducted an in-depth analysis of the expression of CXC chemokines in LUAD and evaluated their potential as therapeutic targets and prognostic biomarkers. The aim of this study was to provide further information to aid clinicians in choosing the appropriate therapeutic drug targets and to identify prognostic biomarkers that can accurately predict the long-term prognosis of patients with LUAD.

\section{Methods}

\section{Analysis of Differential Expression of CXC Chemokines in LUAD}

The Tumor Immune Estimation Resource (TIMER; https://cistrome.shinyapps.io/timer/) is a powerful genome-wide expression platform. ${ }^{10}$ Data were extracted to compare the transcriptome expression profile of the CXC chemokines in lung cancer. A $P$ value $<0.05$ was set as significance selection thresholds.

We also investigated the expression of CXC chemokines in LUAD and normal tissues through the UALCAN database. UALCAN (http://ualcan.path.uab.edu/analysis.html) is a comprehensive web resource for analyzing TCGA, MET500, and the Clinical Proteomic Tumor Analysis Consortium (CPTAC). ${ }^{11}$ Data from TCGA database were obtained using the "Expression Analysis" module and the "LUAD" dataset in UALCAN.

\section{Clinicopathological Parameters and Prognostic Analysis}

Gene Expression Profiling Interactive Analysis (GEPIA) (http://gepia.cancer-pku.cn/index.htm) is a user-friendly analysis tool, which contains RNA sequencing expression data from TCGA and Genotype-Tissue Expression (GTEx), which together comprise 9736 tumor and 8587 normal samples. ${ }^{12}$ We used the "Single Gene Analysis" module of GEPIA to analyze the differential mRNA expression of CXC chemokines in tumor and normal tissues, the pathological stages, and the prognosis of CXC chemokines in LUAD. The "Multiple Gene Comparison" module and the "LUAD" dataset from GEPIA were then used to perform multiple gene comparison analyses. Kaplan-Meier curves were applied to display disease-free survival (DFS) and overall survival (OS). Patients with LUAD were divided into high- and low-expression groups based on median RNA sequencing (RNA-seq) expression value transcripts per million (TPM), and data were validated by hazard ratio (HR) and 95\% confidence interval (CI). A Log rank test was used to determine the statistical difference between two groups. 


\section{Analysis of Genetic Alteration of CXC Chemokines in Patients with LUAD}

The cBioPortal (www.cbioportal.org) is a high-throughput open-access dataset that can visualize multi-dimensional analyses. ${ }^{13}$ Based on the TCGA database, we evaluated genomic alterations related to CXC chemokines in LUAD to explore thei rrole in tumorigenesis.

\section{Analysis of Protein-Protein Interaction Networks of CXC Chemokines}

GeneMANIA (http://www.genemania.org) is a widely used gene prediction server that can offer a systematic and comprehensive prediction of biological network integration. ${ }^{14}$

Search Tool for the Retrieval of Interacting Genes/Proteins (STRING; https://string-db.org) is a database used to analyze the functional protein interaction networks, including physical and functional association. ${ }^{15}$ The predicted protein-protein interaction (PPI) was generated using these interacting genes web databases (GeneMANIA and STRING).

\section{Gene Ontology and Pathway Enrichment Analysis}

Web-Based Set Analysis Toolkit (WebGestalt; http://www.webgestalt.org/option.php) is an intuitive tool that provides functional enrichment analysis for the gene list. ${ }^{16}$ In our study, we set "over-representation analysis" (ORA) as our interested method and the "geneontology and pathway" as a functional database to verify the enrichment of CXC chemokines.

\section{Analysis of Regulatory Factor Networks of CXC Chemokine Gene Expression}

LinkedOmics (http://www.linkedomics.org) is a publicly available portal that provides multiomics data analysis of 32 TCGA cancer types. ${ }^{17}$ The "LinkInterpreter" module from LinkedOmics was applied to conduct the kinase target enrichment and miRNAtarget enrichment analysis through Gene Set Enrichment Analysis (GSEA). The rank criterion was false discovery rate $(\mathrm{FDR})<0.05$, and the simulations were 1000 .

Transcriptional Regulatory Relationships Unraveled by Sentence-based Text mining (TRRUST; https://cistrome. shinyapps.io/timer/) is a reliable database for information on transcriptional regulatory networks, which contains 8444 and 6552 transcriptional factor (TF)-target regulatory relationships of 800 human TFs and 828 mouse TFs, respectively. ${ }^{18}$

\section{Analysis of Immune Infiltration in the Tumor Microenvironment}

TIMER is a publicly available portal tool that provides a systematic analysis of immune infiltration in many types of tumors. We can use the TIMER algorithm online to estimate the immune characteristics of the tumor. In our present study, the "Gene" module was used to evaluate the relationship between immune cell infiltration (B cells, $\mathrm{CD}^{+}{ }^{+} \mathrm{T}$ cells, $\mathrm{CD} 8^{+} \mathrm{T}$ cells, macrophages, neutrophils, and dendritic cells) and CXC chemokine levels in the LUAD dataset. Using the "survival module" data, the Cox proportional hazard model was performed to correct the confounding factors and assess the respective correlations between the clinical prognosis of patients with LUAD and CXC chemokine expression, immune cell infiltration. The Cox's regression model was fitted by function coxph() from R package "survival" in TIMER. The coefficient coef reads as a regression coefficient. The hazard ratio was showed as HR, and its lower and upper 95\% confidential interval are showed in 95\% CI_l and 95\% CI_u.

\section{RNA Sequencing Expression of CXCLI7 Was Measured at the Single Cell Level}

PanglaoDB, a user-friendly single-cell sequencing datasets that allowed users query and explore cell types, genetic pathways and community-curated cell-type marker compendium based on single cell clusters. ${ }^{19}$ We applied the "Sample" module to retrieve the datasets in PanglaoDB. "Human" and "tissue" were set as filter protocols. Homo lung tissues (SRA640325;SRS2769051) which contained 11,186 cells were chose to performed single cell analysis. 


\section{Statistical Analysis}

Differences were analyzed using Student's $t$-test, and correlations were analyzed using Spearman correlation coefficient. Kaplan-Meier curves were applied to display DFS and OS as separated by TPM.A Log rank test was used to determine the differences between the groups. Cox proportional hazard models were used to correct for confounding factors in the analysis of immune-related clinical outcomes. $P$ value $<0.05$ and expressed gene ranking in the top $10 \%$ were set as our significance selection thresholds.

\section{Results}

\section{Dysregulated Expression of CXC Chemokines in Patients with LUAD}

The TIMER database identified aberrant expression of 13 CXC chemokines in LUAD. As shown in Figure 1, 515 lung adenocarcinoma and 59 normal tissues specimens were investigated. The transcriptional levels of CXCL2, CXCL3, CXCL5, CXCL12, and CXCL16 in lung cancer tissues were significantly lower than those in normal tissues, while CXCL9, CXCL10, CXCL13, and CXCL14 were significantly elevated in lung cancer tissues.

To verify above results, the expression of CXC chemokines were also investigated in LUAD and normal tissues via the UALCAN database. As expected, the transcriptional levels of CXCL2 $(P=5.65 \mathrm{e}-5)$, CXCL3 $\left(P=7.45 \mathrm{e}^{-4}\right)$, CXCL11 $(P=1.20 \mathrm{e}-4)$, CXCL12 $(P=1.62 \mathrm{e}-12)$, CXCL16 $(P=1.62 \mathrm{e}-12)$, and CXCL17 $(P=8.82 \mathrm{e}-3)$ were significantly decreased in LUAD, while the expression levels of CXCL6 $(P=1.76 \mathrm{e}-4)$, CXCL9 $(P=7.22 \mathrm{e}-14)$, CXCL10 $(P=3.65 \mathrm{e}-8)$, CXCL13 $(P=2.44 \mathrm{e}-3)$, and CXCL14 $(P=1.11 \mathrm{e}-16)$ were significantly elevated (Figure 2).

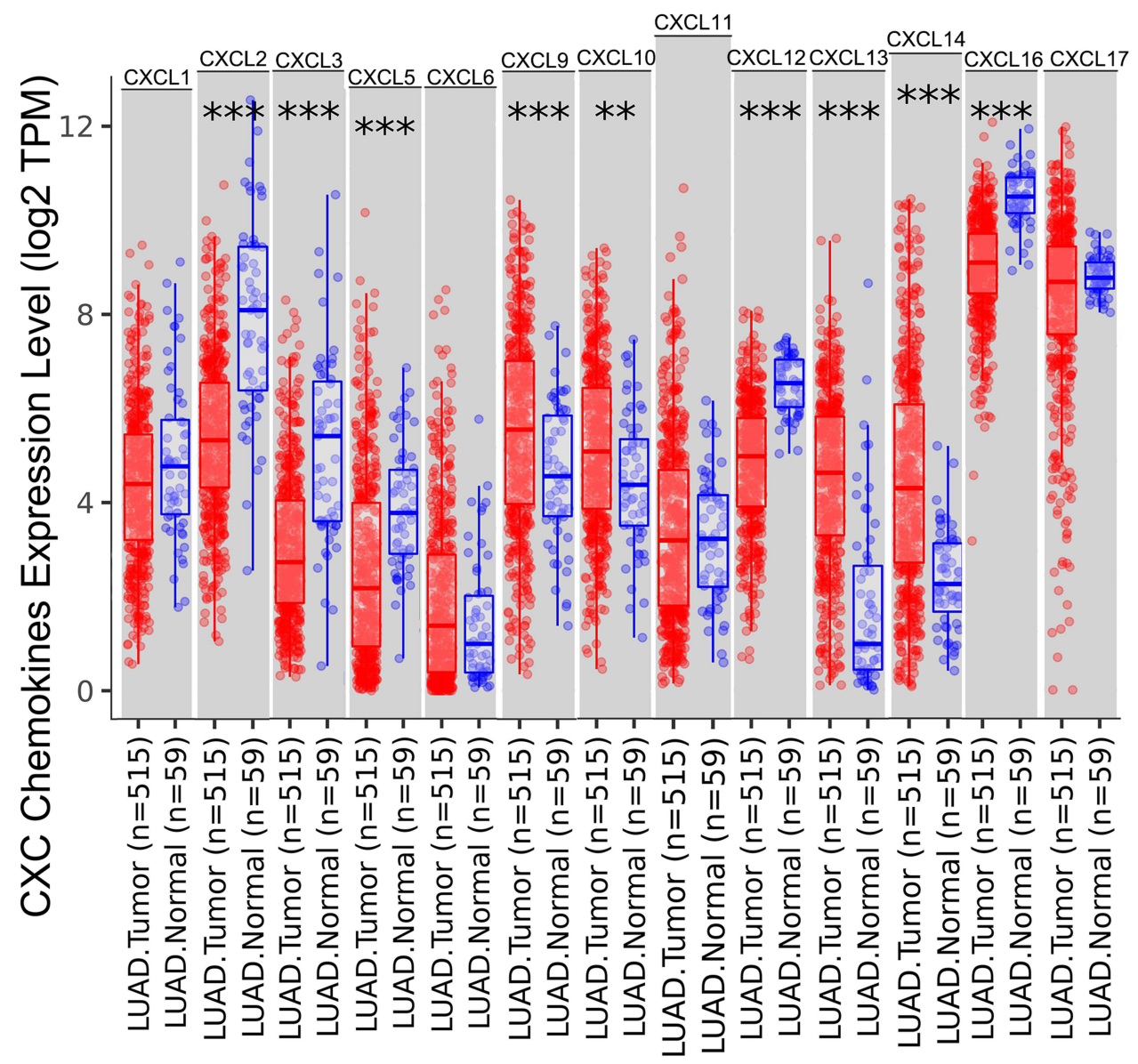

Figure I The expression levels of CXC chemokines in LUAD and normal tissues. The expression status of the CXC chemokines in LUAD and normal tissues was analyzed through TIMER2. **P $<0.01$; ***P $<0.001$.

Abbreviations: CXC, cysteine-X (any amino acid)-cysteine motif; mRNA, messenger ribonucleic acid. 

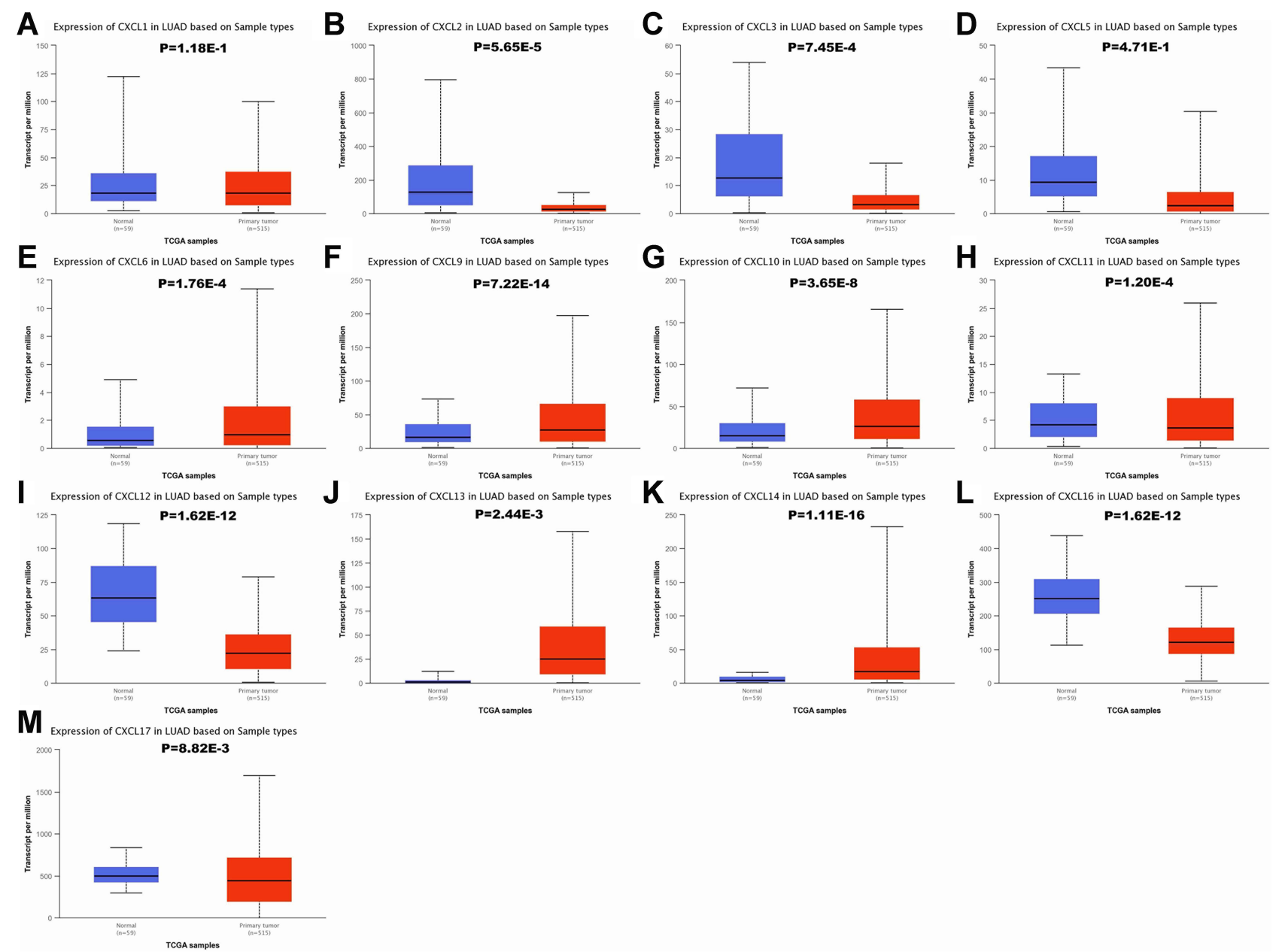

Figure 2 The mRNA expression of CXC chemokines in LUAD tissues and adjacent lung tissues. The transcriptional levels of $C X C L 6 / 9 / / 0 / / 3 / / 4$ were found to be overexpressed in LUAD tissues compared to normal samples (E-G, J and $\mathbf{K}$ ), whereas the expression level of CXCL 2/3/I I/I2/I6/I7 was lower in LUAD tissues than in normal lung tissues (B, C, H, I, L and $\mathbf{M}$ ). There were no significant differences in CXCLI/5 mRNA expression between tumor tissues and normal lung tissues (A and $\mathbf{D})$. The $P$ value threshold was set at 0.05 .

Abbreviations: CXC, cysteine-X (any amino acid)-cysteine motif; CXCL, cysteine-X (any amino acid)-cysteine motif chemokine ligand; LUAD, lung adenocarcinoma.

To identify the relationship between CXC chemokines and occurrence, development, and clinical prognosis of lung adenocarcinoma, we excluded CXCL 8 from further analysis because there was no statistical difference in the transcriptional levels of CXCL 8 between the normal and cancer tissues, according to our previous conclusions. We then evaluated the correlation between the expression of CXC chemokines (CXCL1, CXCL2, CXCL3, CXCL4 (also called PF4; Platelet factor 4 (PF4) is a member of CXC-chemokine family produced by megakaryocytic lineage and stored in platelet $\alpha$-granules.), CXCL5, CXCL6, CXCL7 (also called PPBP; Pro-Platelet Basic Protein (PPBP) is a platelet-derived growth factor that belongs to the CXC chemokine family.), CXCL9, CXCL10, CXCL11, CXCL12, CXCL13, CXCL14, CXCL16, and CXCL17) and the pathological stage of patients with LUAD. We found that aberrant expression of CXCL2 $(P=0.0017)$, CXCL13 $(P=0.0271)$, CXCL16 $(P=0.016)$, and CXCL17 $(P=2.14 \mathrm{e}-5)$ was closely related to tumor pathological stage (Figure 3). This inferred that the abnormal expression of CXCL2, CXCL13, CXCL16, and CXCL17 may be involved in tumor formation, invasion, and metastasis, and could lead to poor clinical prognosis.

\section{The Prognosis Analysis of CXC Chemokines in LUAD Patients}

The Kaplan-Meier plot was applied to the living survival data in the GEPIA dataset to display the DFS and OS in determining the independent prognostic value of the CXC family members in LUAD. Groups were separated by median RNA-seq expression value transcripts per million. A log-rank $P$ value $<0.05$ was considered statistically significant, and 


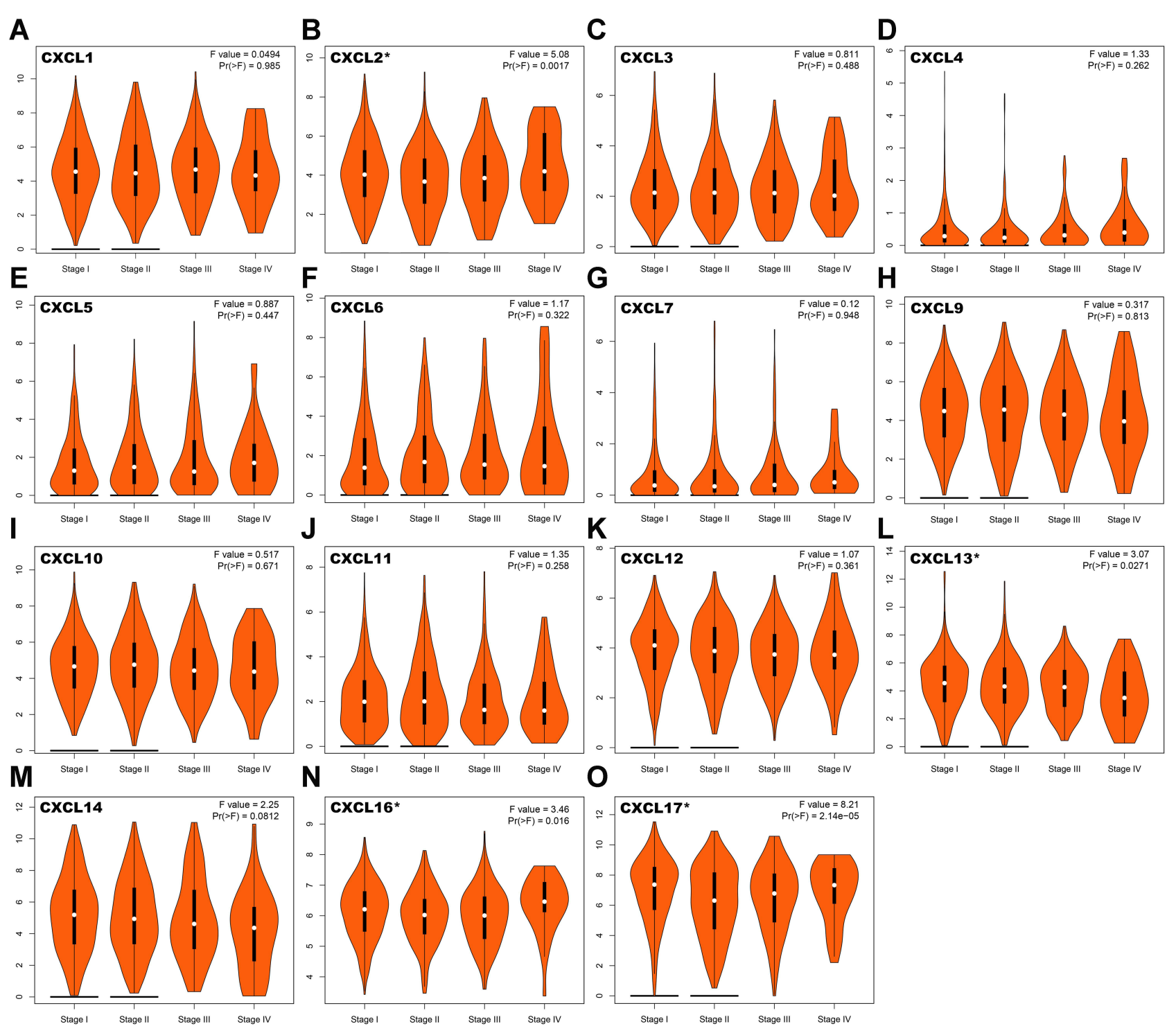

Figure 3 Correlation between the expression of CXC chemokines and tumor stage in LUAD patients. The correlation between the expression of $(\mathbf{A}) \mathrm{CXCLI}$, (B) CXCL2, (C) CXCL3, (D) CXCL4, (E) CXCL5, (F) CXCL6, (G) CXCL7, (H) CXCL9, (I) CXCLI0, (J) CXCLII, (K) CXCLI2, (L) CXCLI3, (M) CXCLI4, (N) CXCLI6 and (O) CXCLI7 and the pathological stage of patients with LUAD. $* P<0.05$.

Abbreviations: CXC, cysteine-X (any amino acid)-cysteine motif; LUAD, lung adenocarcinoma.

a 95\% confidential interval (CI) was also included. As shown in Figure 4, patients with a low transcriptional level of CXCL2 $(P=0.035)$ and CXCL5 $(P=0.027)$ were significantly associated with longer DFS. We also found that patients with low gene transcription of CXCL $7(P=0.017)$ and high expression of CXCL $17(P=0.00045)$ had a better prognosis in LUAD (Figure 5).

\section{Analysis of Genetic Alteration and PPI Networks in Patients with LUAD}

To further analyze the gene and molecular characteristics of the CXC chemokines family members, the cBioPortal dataset was used to analyze the 466 samples from TCGA. Genetic alterations from the members of the CXC family were as follows: CXCL1, CXCL2, CXCL3, CXCL4, CXCL5, CXCL6, CXCL7, CXCL9, CXCL10, CXCL11, CXCL12, CXCL13, CXCL14, CXCL16, and CXCL17 were altered in 7\%, 6\%, 7\%, 6\%,7\%, 9\%, 7\%, 7\%, 6\%, 6\%, 4\%, 6\%, 5\%, $6 \%$, and $7 \%$ of the LUAD samples, respectively (Figure 6A). In these samples, mRNA amplification was the most common type of gene alteration. 

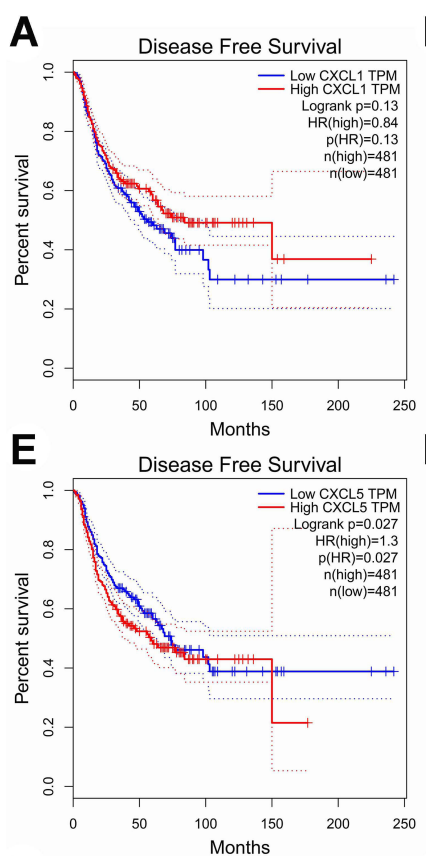

I
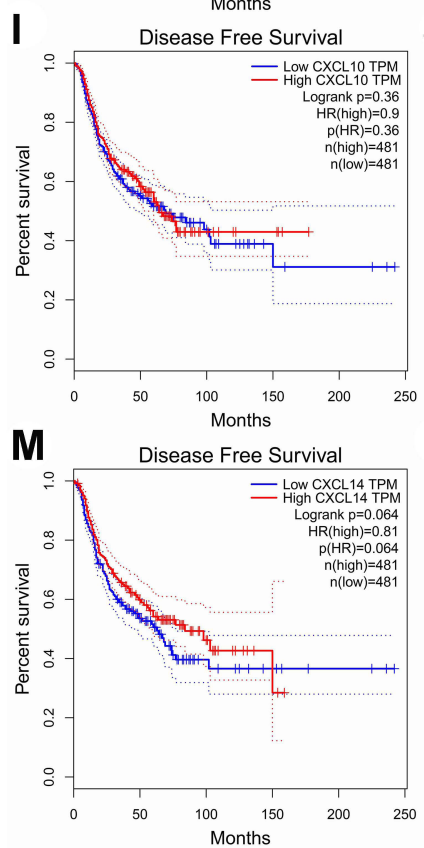

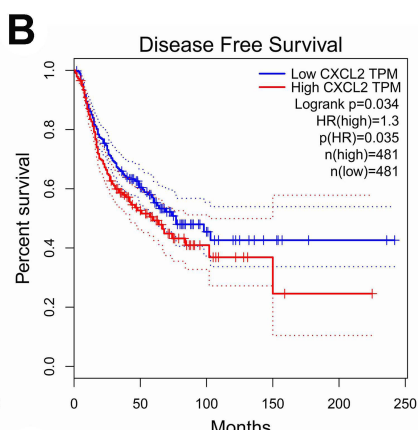

$\mathbf{F}$

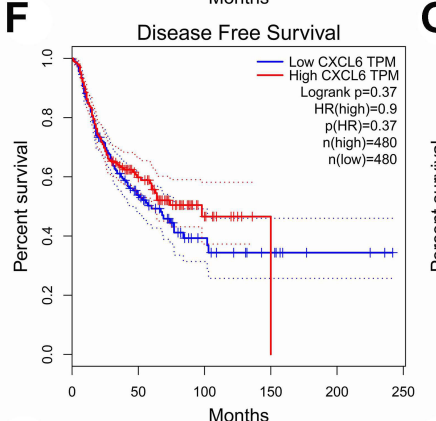

J

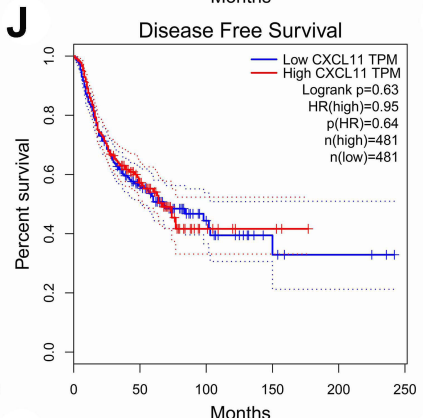

$\mathbf{N}$

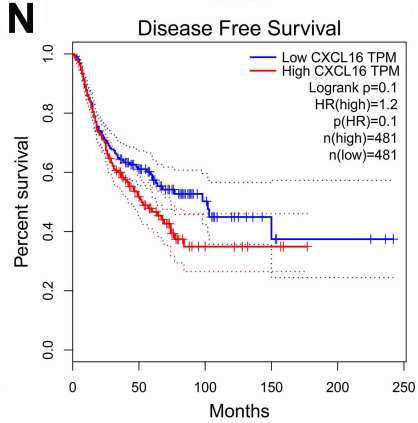

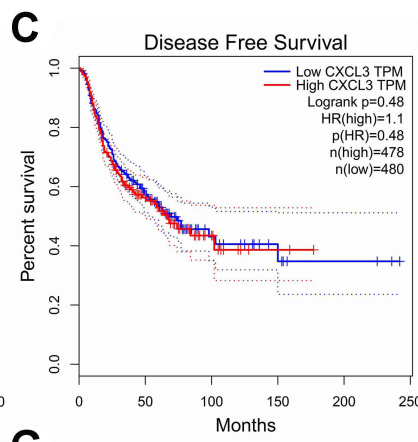

G

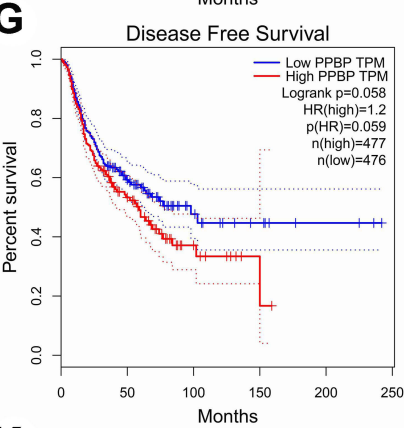

K

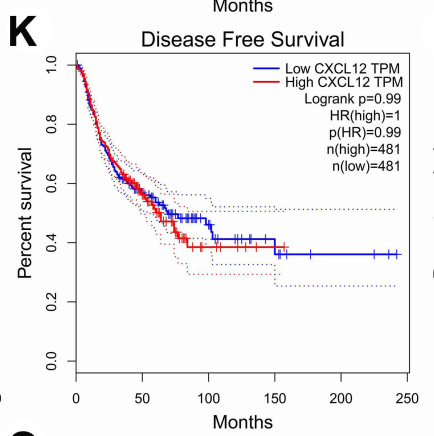

0

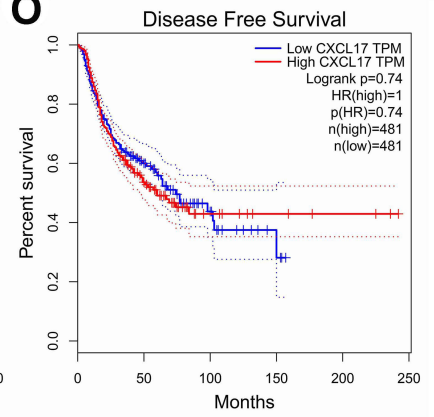

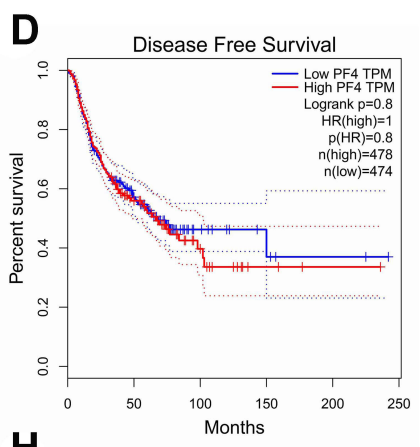

H Disease Free Survival

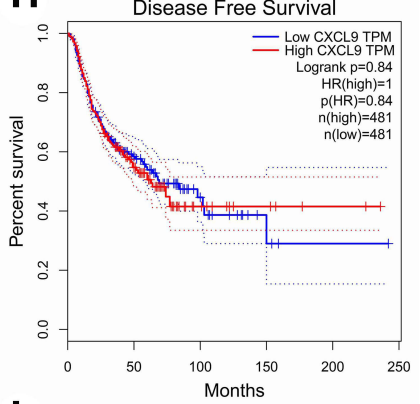

L Disease Free Survival

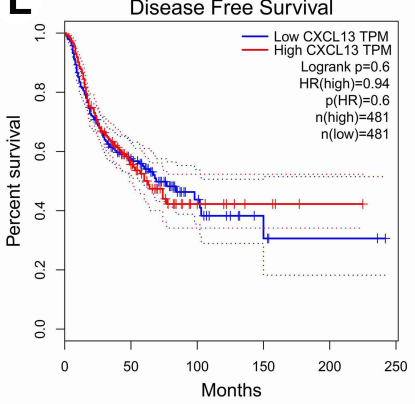

Figure 4 Prognostic value of CXC chemokines in patients with LUAD on the disease-free survival curve. The disease-free survival (DFS) and overall survival (OS)curves comparing patients with high (red) and low (blue) CXC chemokines expressed in LUAD were plotted. DFS curve of (A) CXCLI, (B) CXCL2, (C) CXCL3, (D) CXCL4/PF4, (E) CXCL5, (F) CXCL6, (G) CXCL7/PPBP, (H) CXCL9, (I) CXCLI0, (J) CXCLII, (K) CXCLI2, (L) CXCLI3, (M) CXCLI4, (N) CXCLI6, and (O) CXCLI7 in LUAD. Abbreviations: CXC, cysteine-X (any amino acid)-cysteine motif; LUAD, lung adenocarcinoma.

In addition, STRING was used to perform PPI network analysis of differentially expressed CXC chemokines. The members of the CXC family (CXCL1, CXCL2, CXCL3, CXCL4, CXCL5, CXCL6, CXCL7, CXCL9, CXCL10, CXCL11, CXCL12, CXCL13, CXCL14, CXCL16, and CXCL17) are mainly involved in the chemokine signaling pathway, interleukin 17 (IL-17) signaling pathway, legionellosis, interaction of cytokine-cytokine receptor, and rheumatoid arthritis (Figure 6B). The predicted PPI was also generated by GeneMANIA to evaluate the interactive associations within targeted genes. The highest confidence interaction with an indicated score was selected. The main biological functions of the differentially expressed CXC chemokines were related to cell chemotaxis, chemokine activity, chemokine receptor binding, cytokine activity, and G-protein coupled receptor binding (Figure 6C). 

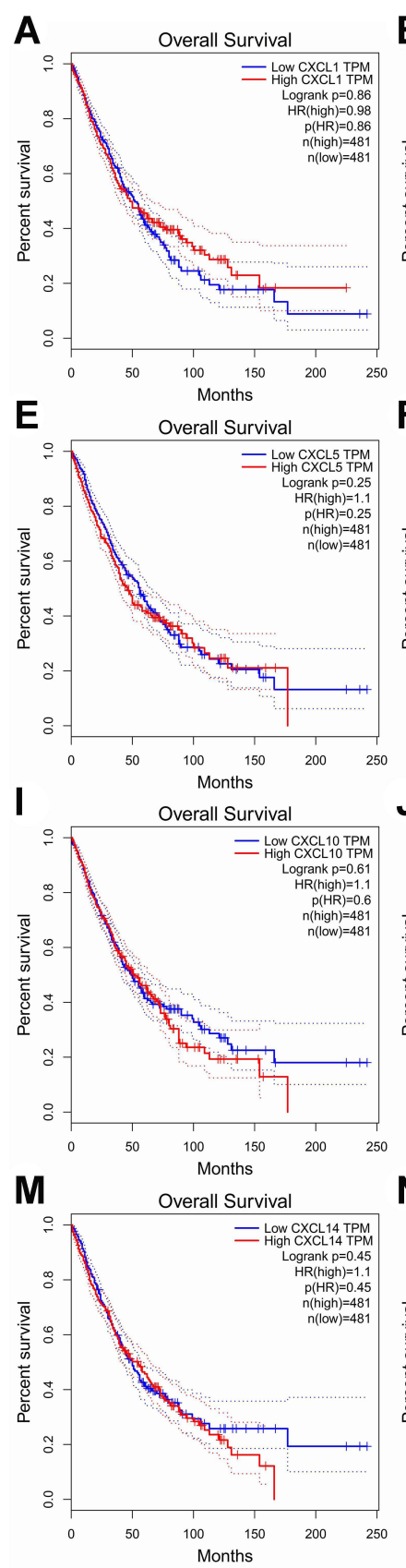
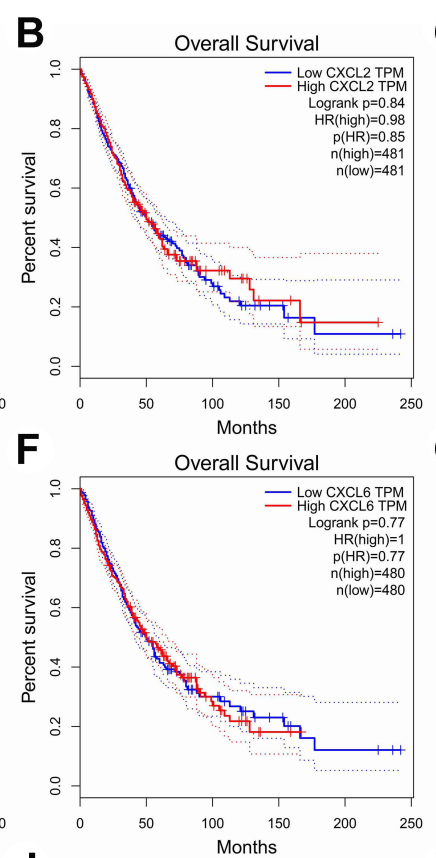

$\mathbf{J}$
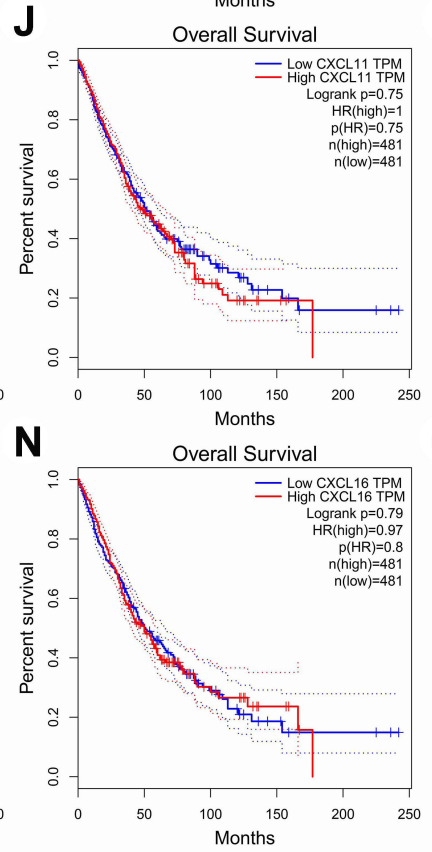
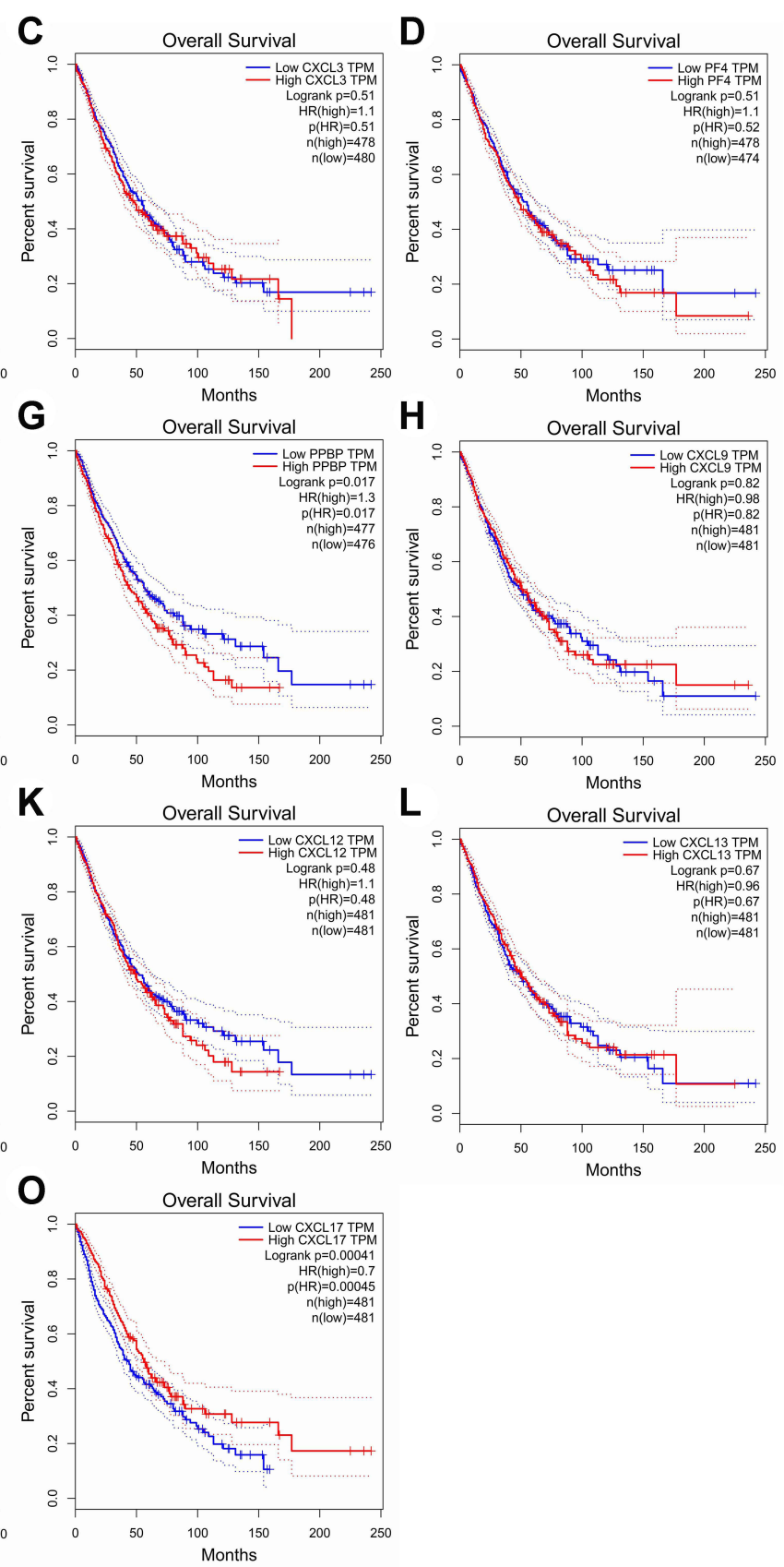

Figure 5 Prognostic feature of CXC chemokines in LUAD patients in the overall survival curve. The overall survival curve of (A) CXCLI, (B) CXCL2, (C) CXCL3, (D) CXCL4/PF4, (E) CXCL5, (F) CXCL6, (G) CXCL7/PPBP, (H) CXCL9, (I) CXCLI0, (J) CXCLII, (K) CXCLI2, (L) CXCLI3, (M) CXCLI4, (N) CXCLI6 and (O) CXCLI7 in LUAD.

Abbreviations: CXC, cysteine-X (any amino acid)-cysteine motif; LUAD, lung adenocarcinoma.

\section{Gene Ontology and Functional Enrichment Analysis of CXC Chemokines}

The WebGestalt database was used to analyze the Gene Ontology (GO) and Kyoto Encyclopedia of Genes and Genomes (KEGG) pathways of members of the CXC chemokine family. Figure 7A-C shows the top-8 most-enriched GO projects. The cellular component (CC) enriched items were extracellular matrix, extracellular space, extracellular region, platelet alpha granule lumen, integral component of plasma membrane, and IL-12 complex. Immune response pathway, cell communication, and signal transduction were related to the occurrence and development of LUAD in the biological process (BP) category. CXC chemokines and their adjacent genes were rich in chemokine receptor binding activity, cytokine receptor binding activity, G-protein coupled receptor activity, and protein-tyrosine kinase activity in molecular 
A

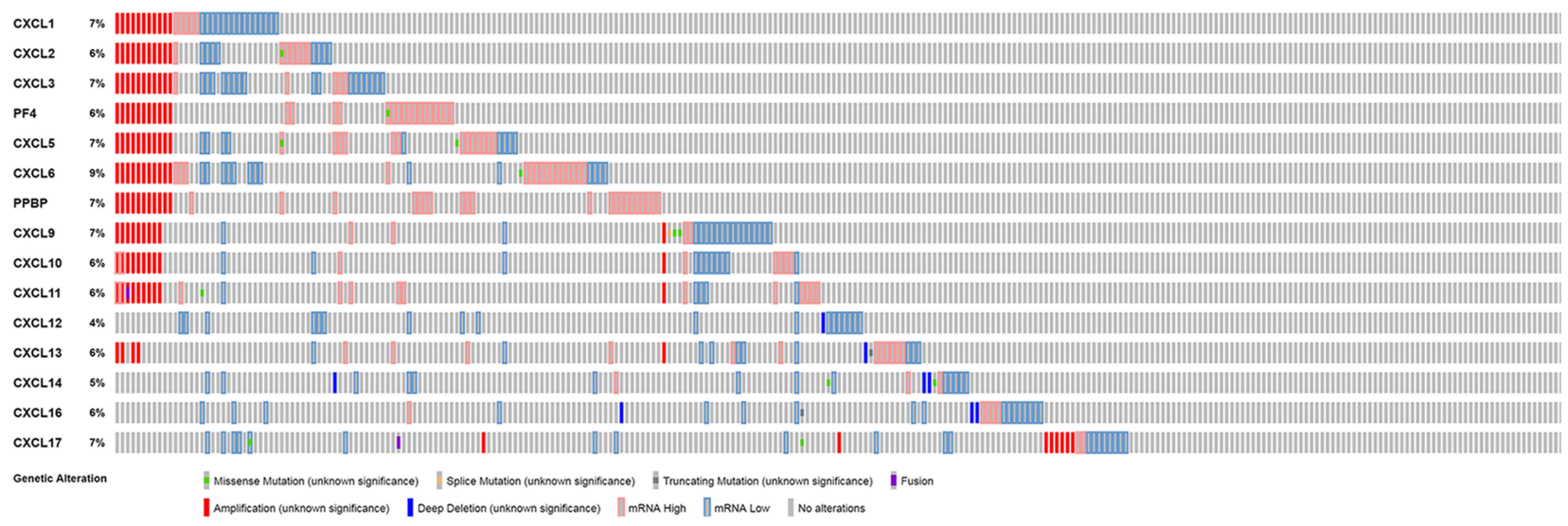

B

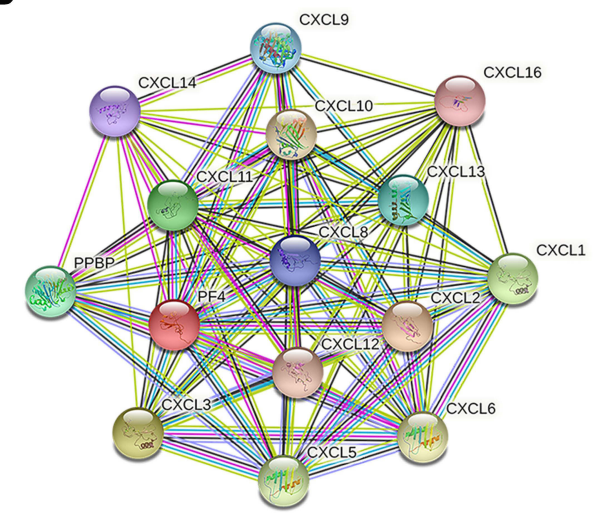

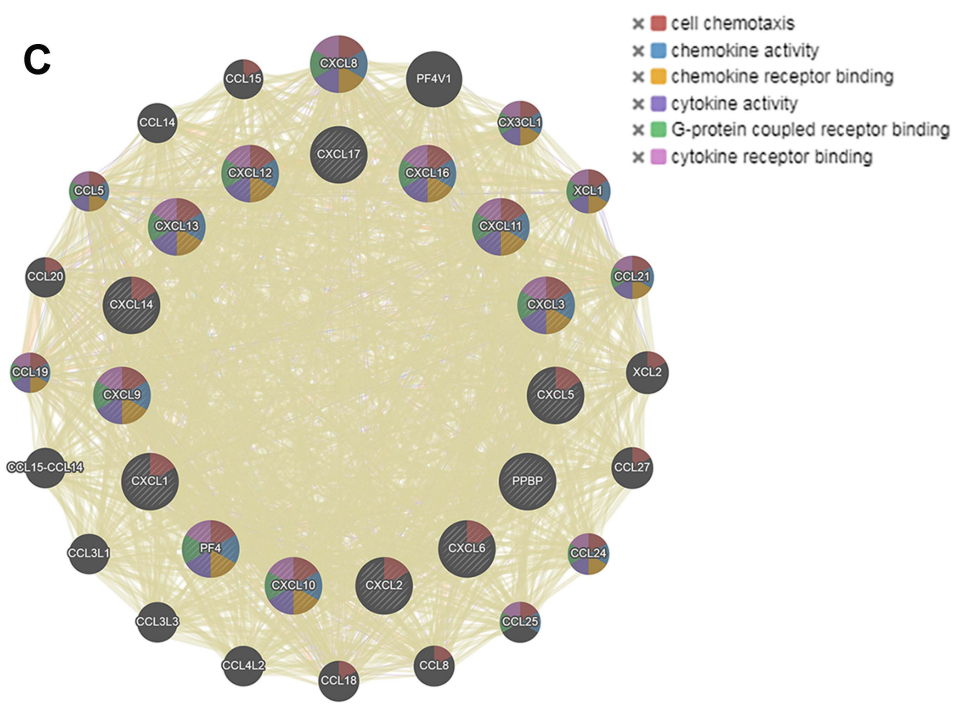

Figure 6 Genetic alteration and protein-protein interaction analyses of the differently expressed CXC chemokines in LUAD patients. (A) Summary of genetic alterations of CXC chemokines in LUAD. (B and C) Protein-protein interaction networks. The nodes represent proteins; the edges represent the interaction of proteins.

Abbreviations: CXC, cysteine-X (any amino acid)-cysteine motif; LUAD, lung adenocarcinoma.

function (MF). For the analysis of the enrichment of the KEGG pathway, the regulatory network of CXC chemokines is shown in Figure 7D. The top 5 KEGG pathways were cytokine-cytokine receptor interaction, chemokine signaling pathway, IL-17 signaling pathway, tumor necrosis factor (TNF) signaling pathway, and rheumatoid arthritis. Some enriched pathways had a significant relationship with the tumorigenesis and development of LUAD.

\section{Regulators of CXC Networks in LUAD}

To explore the possible regulatory factors of the differential expression of CXC chemokines in the lung adenocarcinoma microenvironment, we further analyzed the enrichment of miRNA targets, key transcription-regulated factors, and kinase target networks using the LinkedOmics and the TRRUST database.

Interestingly, not all of the CXC chemokine family members were significantly enriched in miRNA targets (Table 1). The miRNA targets most significantly enriched by the CXCL2 chemokines were -GGCACAT-(FDR=0.01370). -ATAGGAA-, TCCCCAC- and -TACTTGA- might be the miRNA binding sites corresponding to the transcripts of CXCL3 (FDR $=0.02956$ ), CXCL10 (FDR=0.02271), and CXCL17 (FDR=0.01077), respectively. Next, we explored possible key transcriptionregulated factors of the differentially expressed CXC chemokines. CXCL1, CXCL2, CXCL5, CXCL10, CXCL12, and CXCL14 were included as valid query genes in the TRRUST database. As shown in Table 2, v-relreticuloendotheliosis viral oncogene homolog avian (RELA), nuclear factor of kappa light polypeptide gene enhancer in B-cells 1 (NFKB1), and Sp1 

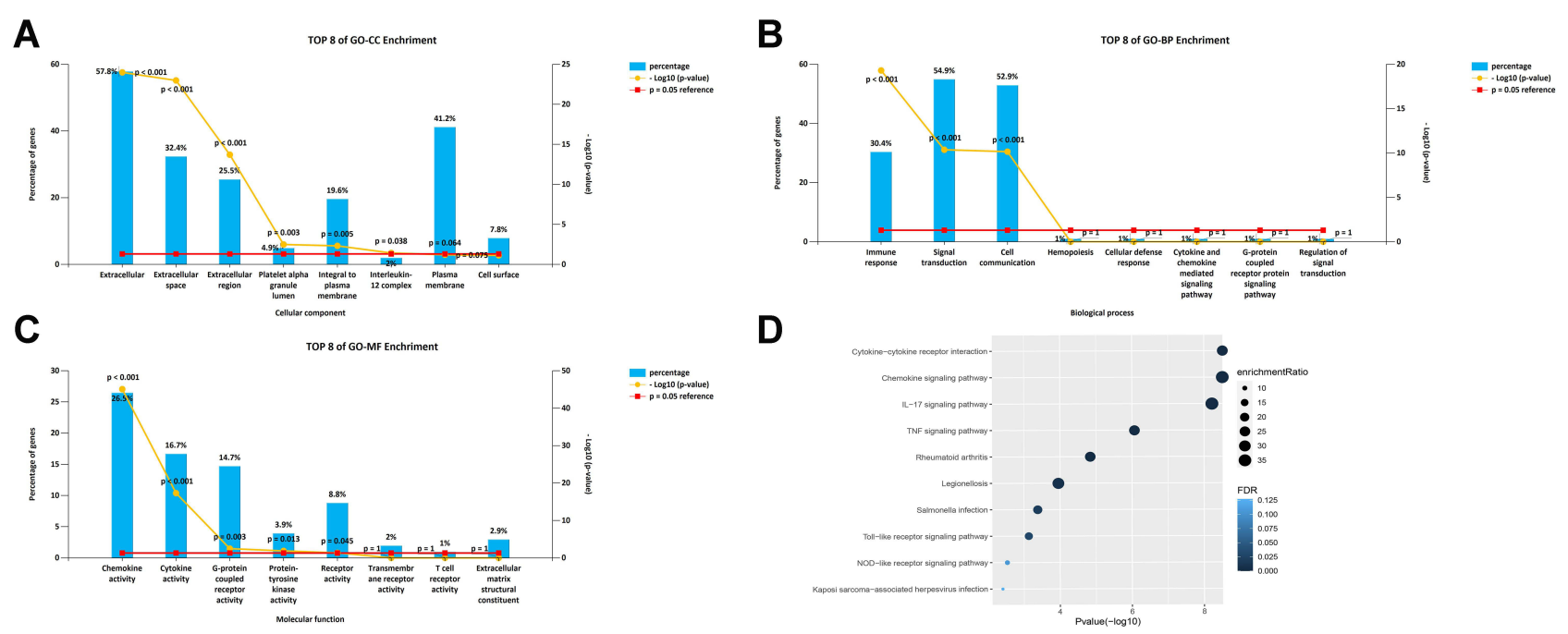

Figure 7 GO and KEGG enrichment analysis of CXC chemokines and their80coexpressed genes. (A-C) Column charts of GO enrichment in the terms of CC, BP, and MF. (D) Volcano plot of KEGG enriched terms.

Abbreviations: CXC, cysteine-X (any amino acid)-cysteine motif; GO, Gene Ontology; KEGG, Kyoto Encyclopedia of Genes and Genomes; CC, cellular component; BP, biological process; MF, molecular function.

transcription factor (SP1) were key transcription-regulated factors of CXC chemokines. RELA $(P=1.16 \mathrm{e}-06)$ and NFkB1 $(P=1.19 \mathrm{e}-16)$ were the key regulated factors in modulating the transcription of CXCL1, CXCL2, CXCL5, CXCL10, and CXCL12. SP1 $(P=0.00369)$ was the key transcription factor for CXCL1, CXCL5, and CXCL14.

Table I The Enrichment of miRNA Targets of the CXC Chemokine Family Members in LUAD

\begin{tabular}{|l|c|c|c|c|}
\hline CXC Chemokines & Gene Set & LEN & NES & FDR \\
\hline CXCLI & CAGCACT, MIR-5I2-3P & 44 & -1.2590 & 0.44720 \\
CXCL2 & GGCACAT, MIR-455 & 6 & 1.9156 & 0.01370 \\
CXCL3 & ATAGGAA, MIR-202 & 32 & -1.9187 & 0.02956 \\
CXCL5 & TTGCCAA, MIR-I82 & 75 & 1.5979 & 0.08137 \\
CXCL6 & ACGCACA, MIR-2I0 & 2 & -1.4800 & 0.32184 \\
CXCL9 & TCGATGG, MIR-2I3 & 1 & 1.4539 & 0.23308 \\
CXCLI0 & TCCCCAC, MIR-49I & 18 & -1.9132 & 0.02271 \\
CXCLII & TCGATGG, MIR-2I3 & 1 & 1.4554 & 0.27673 \\
CXCLI2 & CGTCTTA, MIR-208 & 2 & 1.3647 & 0.43778 \\
CXCLI3 & CCACACA, MIR-I47 & 19 & -1.4645 & 0.20960 \\
CXCLI4 & ACCGAGC, MIR-423 & 2 & -1.1603 & 0.62911 \\
CXCLI6 & GCAAGAC, MIR-43I & 9 & 1.6370 & 0.20920 \\
CXCLI7 & TACTTGA, MIR-26A, MIR-26B & 95 & -1.8714 & 0.01077 \\
\hline
\end{tabular}

Abbreviations: miRNA, microRNA; CXC, cysteine-X (any amino acid)-cysteine motif; LUAD, lung adenocarcinoma; LEN, leading edge number; NES, normalized enrichment score; FDR, false discovery rate.

Table 2 Key Transcription Regulated Factors of the Differentially Expressed CXC Chemokines in LUAD

\begin{tabular}{|l|c|c|c|}
\hline Key TF & Regulated Gene & P-value & FDR \\
\hline RELA & $C X C L I, C X C L 2, C X C L 5, C X C L 10, C X C L I 2$ & $1.16 \mathrm{e}-06$ & $1.79 \mathrm{e}-06$ \\
NFKBI & $C X C L I, C X C L 2, C X C L 5, C X C L 10, C X C L I 2$ & $1.19 \mathrm{e}-16$ & $1.79 \mathrm{e}-06$ \\
SpI & $C X C L I, C X C L 5, C X C L / 4$ & 0.00369 & 0.00369 \\
\hline
\end{tabular}

Abbreviations: CXC, cysteine-X (any amino acid)-cysteine motif; LUAD, lung adenocarcinoma; TF, transcription factor; RELA, v-relreticuloendotheliosis viral oncogene homolog avian; NFKBI, nuclear factor of kappa light polypeptide gene enhancer in B-cells I; SPI, SpI transcription factor; FDR, false discovery rate. 
We then identified the most statistically significant kinase targets for CXC chemokines using the LinkedOmics database (Table 3). TTK was suggested as a target for the CXCL1 kinase target network. PLK1 was primarily associated with CXCL2. Components of the CXCL3 kinase target network were mainly associated with ATM. PAK2 and LRRK2 were the main targets in the CXCL5 and CXCL6 kinase target networks, respectively. ITK was suggested as the kinase target for CXCL9. LCK, FGR, and MAP3K3 were primarily related to CXCL10, CXCL11, and CXCL12, respectively. The CXCL13, CXCL14, CXCL16, and CXCL17 kinase target networks were related to the PIM1, SYK, PLK3, and CHEK2, respectively.

\section{Correlation Analysis Between CXC Chemokines and the 6 Main Infiltrating Immune Cells}

CXC chemokines mediate the inflammatory response and inflammatory cell infiltration in the tumor microenvironment to affect immune evasion, invasion, and metastasis of the tumor, thus affecting the prognosis of patients. Therefore, the TIMER database was used to conduct a comprehensive analysis of the differential expression of the CXC chemokines and infiltrating immune cells (B cells, CD8+ T cells, CD4+ T cells, macrophages, neutrophils, and dendritic cells). The analysis showed that the expression of CXCL1 was positively correlated with the infiltration of neutrophils (Cor $=0.301, P=1.45 \mathrm{e}$ -11 ) and the infiltration of dendritic cells (Cor $=0.09, P=4.75 \mathrm{e}-2$; Figure $8 \mathrm{~A}$ ). There was a positive relationship between CXCL2 expression and neutrophils infiltration ( $\mathrm{Cor}=0.182, P=5.53 \mathrm{e}-5$; Figure $8 \mathrm{~B}$ ). The expression of CXCL3 expression was positively associated with macrophage infiltration $(\mathrm{Cor}=0.078, P=8.58 \mathrm{e}-2)$ and infiltration of neutrophils (Cor $=0.257$, $P=1.07 \mathrm{e}-8$; Figure $8 \mathrm{C}$ ). There was positive correlation between the expression of CXCL5 and the infiltration of macrophages $(\mathrm{Cor}=0.13, P=4.21 \mathrm{e}-3)$ and neutrophils $(\mathrm{Cor}=0.22, P=1.07 \mathrm{e}-6)$ and a negative correlation between CXCL5 expression and $\mathrm{CD}^{+} \mathrm{T}$ cell infiltration (Cor $\left.=-0.105, P=2.05 \mathrm{e}-2\right)$ and $\mathrm{B}$ cells $(\mathrm{Cor}=-0.178, P=8.31 \mathrm{e}-5$; Figure $8 \mathrm{D})$. The expression of CXCL6 and the infiltration of $\mathrm{CD}^{+} \mathrm{T}$ cells $(\mathrm{Cor}=0.103, P=2.37 \mathrm{e}-2)$, macrophages (Cor $=0.125$, $P=5.81 \mathrm{e}-3$ ), neutrophils (Cor=0.267, $P=3.34 \mathrm{e}-9$ ), and dendritic cells ( $\mathrm{Cor}=0.167, P=2.08 \mathrm{e}-4$; Figure $8 \mathrm{E}$ ) was positively correlated. There was positive correlation between CXCL7 expression and the infiltration of macrophages (Cor $=0.143$, $P=1.54 \mathrm{e}-3)$ and neutrophils ( $\mathrm{Cor}=0.127, P=5.13 \mathrm{e}-3$ ), and there was a negative correlation between the expression of CXCL7 and the infiltration of CD4 ${ }^{+}$T cells (Cor $\left.=-0.123, P=6.55 \mathrm{e}-3\right)$ and $\mathrm{B}$ cells ( $\mathrm{Cor}=-0.218, P=1.33 \mathrm{e}-6$; Figure $8 \mathrm{~F}$ ). Similarly, the expressions of CXCL9, CXCL10, CXCL11, and CXCL12 were positively associated with the infiltration of $\mathrm{B}$ cells, $\mathrm{CD} 8^{+} \mathrm{T}$ cells, $\mathrm{CD} 4^{+} \mathrm{T}$ cells, macrophages, neutrophils, and dendritic cells (all $P<0.05$; Figure 8G-J). Except for macrophages, CXCL13expression was positively correlated with the infiltration all immune cell types (B cells, CD $8^{+}$ T cells, $\mathrm{CD}^{+}$T cells, neutrophils, and dendritic cells; all $P$ values $<0.05$;Figure $\left.9 \mathrm{~K}\right)$. The expression of CXCL14 and the infiltration of $\mathrm{CD}^{+}$T cells $(\mathrm{Cor}=0.229, P=3.63 \mathrm{e}-7)$, B cells $\left(\mathrm{Cor}=0.221, P=8.84 \mathrm{e}^{-7}\right)$ and dendritic cells $(\mathrm{Cor}=0.191, P=$

Table 3 The Kinase Target Networks of CXC Chemokines in LUAD

\begin{tabular}{|l|c|c|c|c|c|}
\hline CXC Chemokines & Gene Set & Description & LEN & NES & FDR \\
\hline CXCLI & Kinase_TTK & TTK protein kinase & 2 & $-2.075 I$ & 0.0054 \\
CXCL2 & Kinase_PLKI & Polo like kinase I & 44 & -2.1866 & 0 \\
CXCL3 & Kinase_ATM & ATM serine/theronine kinase & 43 & -2.0522 & 0.0040 \\
CXCL5 & Kinase_PAK2 & P2I(RACI)actived kinase 2 & 1 I & 1.6583 & 0.3895 \\
CXCL6 & Kinase_LRRK2 & Leucine rich repeat kinase 2 & 7 & -2.0165 & 0.0110 \\
CXCL9 & Kinase_ITK & IL2 inducible T-cell kinase & 1.4877 & 0.1712 \\
CXCLI0 & Kinase_LCK & LCK proto-oncogene, Src family tyrosine kinase & 20 & 1.7554 & 0 \\
CXCLII & Kinase_FGR & FGR proto-oncogene, Src family tyrosine kinase & 4 & 1.4993 & 0.2898 \\
CXCLCI2 & KinaseMAP3K3 & Mitogen-actived protein kinase kinasekinase 3 & 1 & 1.4348 & 0.1493 \\
CXCL13 & Kinase_PIMI & Pim-I pro-oncogene, serine/threonine kinase & 3 & 1.4908 & 0.2044 \\
CXCL14 & Kinase_SYK & Spleen associated tyrosine kinase & 20 & 1.8864 & 0 \\
CXCL16 & Kinase_PLK3 & Polo like kinase 3 & 8 & -1.9780 & 0 \\
CXCLI7 & Kinase_CHEK2 & Checkpoint kinase 2 & 13 & -2.0167 & 0 \\
\hline
\end{tabular}

Abbreviations: CXC, cysteine-X (any amino acid)-cysteine motif; LUAD, lung adenocarcinoma; LEN, leading edge number; NES, normalized enrichment score; FDR, false discovery rate. 

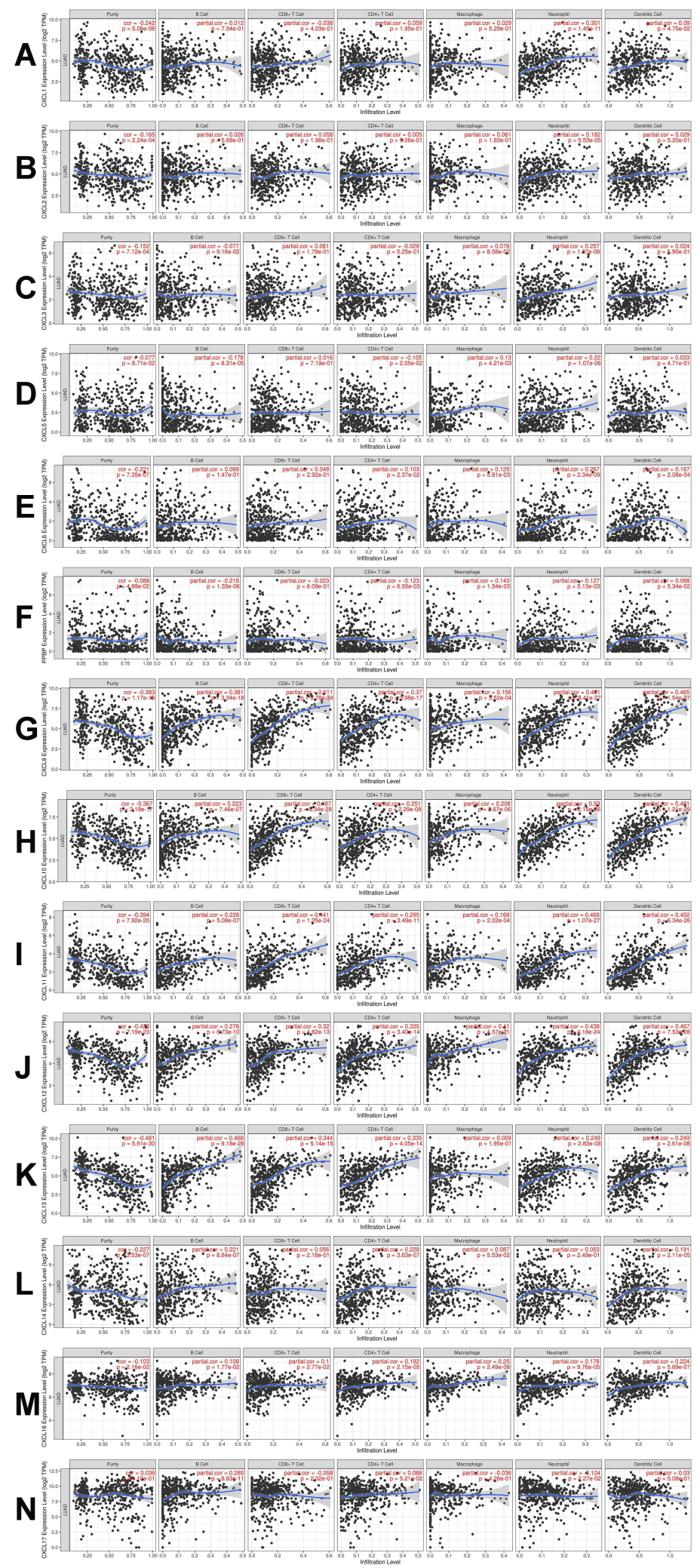

Figure 8 The correlation between CXC chemokines and the 6 main infiltrating immune cells. The correlation between the abundance of 6 immune cells and the expression of (A) CXCLI, (B) CXCL2, (C) CXCL3, (D) CXCL5, (E) CXCL6, (F) CXCL7, (G) CXCL9, (H) CXCLI0, (I) CXCLII, (J) CXCLI2, (K) CXCLI3, (L) CXCLI4, (M) CXCLI6, and (N) CXCLI7 in LUAD.

Abbreviations: CXC, cysteine-X (any amino acid)-cysteine motif; LUAD, lung adenocarcinoma. 


\section{Lung cells (SRA640325:SRS2769051)}
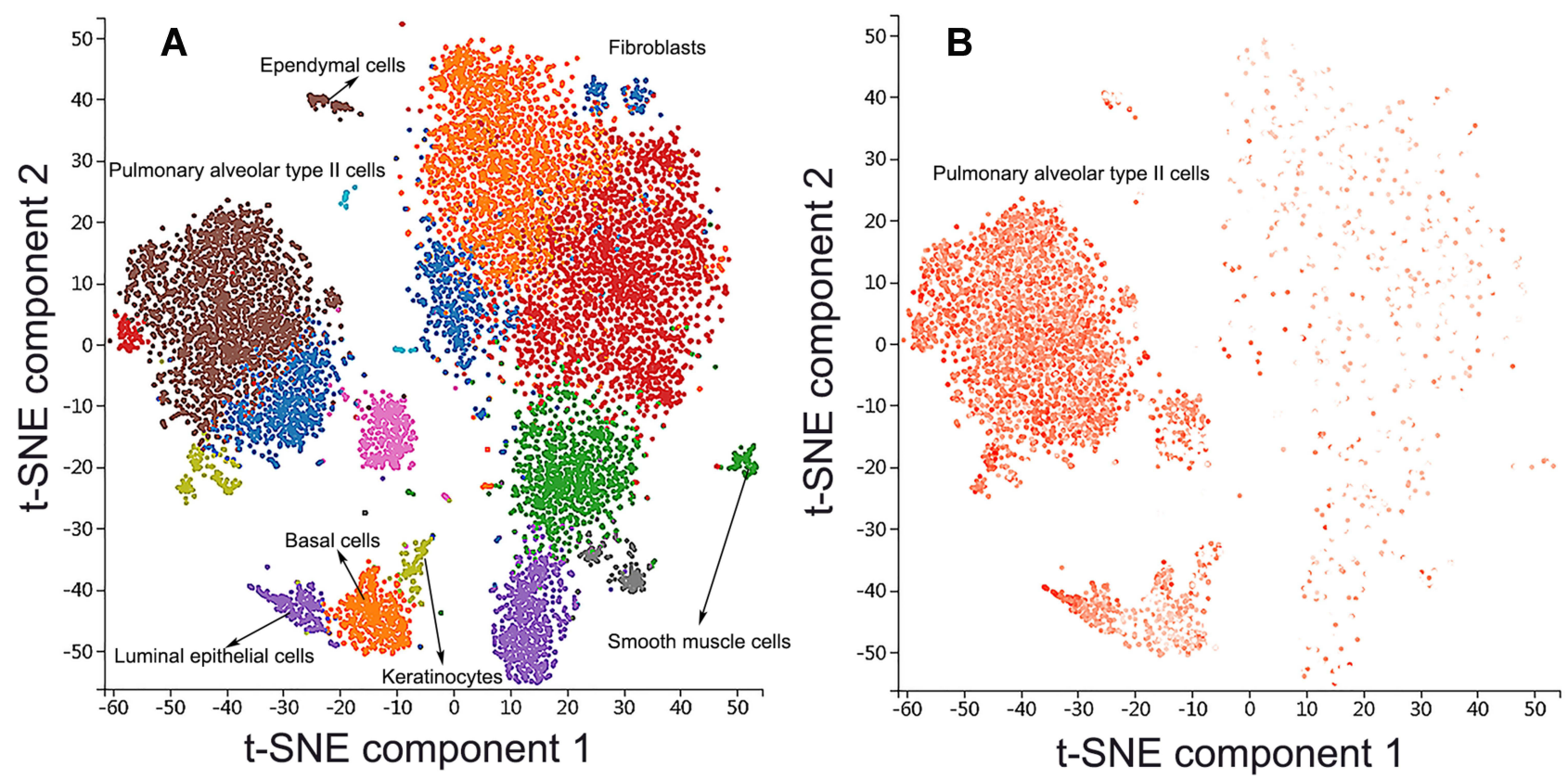

Figure 9 Single-cell RNA sequencing localization analysis of CXCLI7. (A) Lung tissue cells were divided into 7 cell clusters. (B) CXCLI7 was enriched in pulmonary alveolar type II cell clusters.

2.11e-5; Figure 8L) was positively correlated. CXCL16 was positively correlated with the infiltration all immune cell types (B cells, $\mathrm{CD}^{+} \mathrm{T}$ cells, $\mathrm{CD} 4^{+} \mathrm{T}$ cells, neutrophils, and dendritic cells; all $P$ values $<0.05$; Figure $8 \mathrm{M}$ ). There was a positive correlation between CXCL17 expression and the infiltration of B cells (Cor $\left.=0.289, P=8.63 \mathrm{e}^{-11}\right)$ and $\mathrm{CD} 4^{+} \mathrm{T}$ cells $($ Cor $=$ $0.088, P=5.21 \mathrm{e}-2)$, and a negative correlation between the expression of CXCL17 and the infiltration of neutrophils (Cor $=$ $-0.104, P=2.27 \mathrm{e}-2$; Figure $8 \mathrm{~N})$.

In addition, the "survival" module was used to explore the clinical outcome in patients with LUAD, and Cox proportional hazard models were conducted to correct for the confounding factors $\left(\mathrm{CD} 4^{+} \mathrm{T}\right.$ cells, $\mathrm{CD} 8^{+} \mathrm{T}$ cells, macrophages, neutrophils, dendritic cells, CXCL1, CXCL 2, CXCL 3, CXCL5, CXCL 6, CXCL7, CXCL 9, CXCL 10, CXCL 11, CXCL 12, CXCL 13, CXCL 14, CXCL 16, and CXCL17).From this, we found that B cell infiltration $(P=0.001$, coef $=-4.48$; Table 4$)$ could prolong the survival of the LUAD patients.

\section{Single-Cell RNA Sequencing Localization Analysis of Prognostic Biomarker Gene}

Prognostic analysis had shown that patients with low gene transcription of CXCL $7(P=0.017)$ and high expression of CXCL $17(P=0.00045)$ had a better prognosis in LUAD. Thus, we chose CXCL 17 as biomarker gene to further analyze the biological mechanism of CXCL17 in the immune microenvironment of lung adenocarcinoma. CXCL7 was excluded because it has no differential expression between LUAD and normal tissues. PanglaoDB dataset was utilized to performed single-cell RNA sequencing localization analysis. As shown in Figure 9A, lung tissue cells were divided into 7 cell clusters included pulmonary alveolar type II cells $(P=1.03 \mathrm{e}-14)$, fibroblasts $(P=2.40 \mathrm{e}-12)$, basal cells ( $P=1.08 \mathrm{e}-11)$, smooth muscle cells $(P=4.35 \mathrm{e}-8)$, ependymal cells $(P=3.47 \mathrm{e}-18)$, keratinocytes $(P=1.43 \mathrm{e}-4)$, luminal epithelial cells $(P=8.43 \mathrm{e}-7)$. Then CXCL17 was set as overlay expression of gene, and we found that CXCL17 was enriched in pulmonary alveolar type II cell clusters according to single-cell analysis (Figure 9B). Thus, we might infer that the reduction of CXCL17 expression in pulmonary alveolar type II cells played an important role in the carcinogenesis of LUAD. 
Table 4 Multivariate Cox Proportional Hazard Model of CXC Chemokines and 6 Infiltrating Immune Cells in LUAD

\begin{tabular}{|l|c|c|c|c|c|}
\hline Variables & Coef & HR & $\mathbf{9 5 \%}$ Cl_I & $\mathbf{9 5 \%}$ CI_u & P-value \\
\hline B cell & -4.482 & 0.011 & 0.001 & 0.176 & 0.001 \\
CD8 ${ }^{+}$T cell & 0.104 & 1.109 & 0.131 & 9.356 & 0.924 \\
CD4 ${ }^{+}$T cell & 2.363 & 10.624 & 0.479 & 235.690 & 0.135 \\
Macrophage & 0.072 & 1.075 & 0.070 & 16.577 & 0.959 \\
Neutrophil & -1.923 & 0.0146 & 0.001 & 19.080 & 0.439 \\
Dendritic & 0.224 & 1.251 & 0.288 & 0.765 \\
CXCLI & -0.031 & 0.969 & 0.824 & 1.140 & 0.707 \\
CXCL2 & -0.078 & 0.925 & 0.785 & 1.090 & 0.353 \\
CXCL3 & 0.217 & 1.243 & 0.981 & 1.574 & 0.071 \\
CXCL5 & 0.036 & 1.037 & 0.941 & 1.143 & 0.465 \\
CXCL6 & 0.010 & 1.010 & 0.877 & 1.164 & 0.887 \\
CXCL9 & -0.006 & 0.994 & 0.798 & 1.239 & 0.958 \\
CXCLI0 & -0.116 & 0.890 & 0.635 & 1.249 & 0.501 \\
CXCLII & 0.195 & 1.215 & 0.927 & 1.594 & 0.159 \\
CXCLI2 & -0.030 & 0.970 & 0.830 & 1.134 & 0.702 \\
CXCLI3 & -0.017 & 0.984 & 0.879 & 1.100 & 0.771 \\
CXCLI4 & 0.045 & 1.046 & 0.978 & 1.119 & 0.193 \\
CXCLI6 & -0.081 & 0.923 & 0.747 & 1.139 & 0.454 \\
CXCLI7 & -0.072 & 0.931 & 0.857 & 1.011 & 0.087 \\
\hline
\end{tabular}

Abbreviations: CXC, cysteine-X (any amino acid)-cysteine motif; LUAD, lung adenocarcinoma; coef, regression coefficient; HR, hazard ratio; 95\% Cl_I and 95\%Cl_u, lower and upper $95 \%$ confidential interval.

\section{Discussion}

In the present study, we showed the aberrant expression profiles of the CXC chemokine family in patients with LUAD. Patients with low gene transcription of CXCL $7(P=0.017)$ and high expression of CXCL $17(P=0.00045)$ had a better prognosis. The enrichment of miRNA targets, key transcription-regulated factors, and kinase target networks were also analyzed to explore regulatory factors of the differential expression of CXC chemokines in lung adenocarcinoma microenvironment. Cox proportional hazard model test was conducted and indicated that $\mathrm{B}$ cell infiltration $(P=0.001$, coef $=-4.48$ ) could prolong the survival of the LUAD patients. CXCL17 was enriched in pulmonary alveolar type II cell clusters. And, the reduction of CXCL17 expression in pulmonary alveolar type II cells played an important role in the carcinogenesis of LUAD.

The long-term inflammatory response can provide an ideal microenvironment for the development and growth of tumor cells, so it is believed to promote carcinogenesis. ${ }^{20}$ As an important component of the chemokine family, CXC chemokines have been revealed to directly affect tumor transformation, survival, growth, invasion, and metastasis, and indirectly affect tumor development by affecting angiogenesis and crosstalk between tumor cells and interstitial cells. ${ }^{21}$

Crosstalk between cancer cells and immune cells is a key part of tumorigenesis, metastasis, and therapeutic effects for patients with malignancies. ${ }^{6}$ Therefore, it is important to identify specific CXC chemokines from the CXC family as precise therapeutic targets and prognostic biomarkers in the immune system.

The results of our study showed that the transcriptional level of CXCL 9/10/13/14 was significantly upregulated, while the transcriptional expression of CXCL 2/3/12/16/17 was lower in LUAD tissues compared to normal tissues. Interestingly, we also found that aberrant expression of CXCL2, CXCL13, CXCL16, and CXCL17 was closely related to the pathological tumor, indicating that these chemokines could promote or inhibit tumor invasion and metastasis by changing microenvironmental tumor mediators. Similarly, $\mathrm{Hu}$ et al found that CXCL2elevation disrupted $\mathrm{CD}^{+} \mathrm{T}$ cell accumulation at tumor sites, causing accelerated tumor progression. ${ }^{22}$ In contrast, Yang et al revealed thatCXCL13 could activate CXCR5 $+\mathrm{CD} 8+\mathrm{T}$ cells infiltration in the MIT, thus inhibiting tumor metastasis. ${ }^{23}$ Ikeda et al also reported that upgraded expression of CXCL16 in the tumor microenvironment could act on MKN45 cells in a paracrine manner. They further induced the expression of ROR1 in MKN45 cells through the CXCR6-STAT3 pathway, thus promoting tumor 
progression. ${ }^{24}$ Matsui et al further revealed that aberrant expression of CXCL17 in tumor cells could promote tumor progression by recruiting immature myeloid-derived cells. ${ }^{25}$ These findings were consistent with our retrieval results.

Our study revealed that the low expression of CXCL7 and high expression of CXCL17 were significantly associated with better OS in LUAD patients, while the expression level of CXCL17 in LUAD tumor tissues was significantly lower than that in nontumor tissues. We also confirmed that CXCL17 expression was significantly associated with tumor stage in patients with LUAD. These findings suggest that CXCL17 could be a prognostic marker and a potential oncogene for LUAD.

Since the differential expression of CXC chemokines in lung cancer tissues was statistically significant, we further explored their molecular characteristics in lung cancer and found that there were frequent genetic alterations in differentially expressed CXC chemokines in LUAD. Among them, mRNA amplification was the most common type. Gene mutations play an important role in the tumorigenesis and progress of lung adenocarcinoma and provide ideas for targeted treatment in lung cancer. ${ }^{26}$

To further explore the important biological function of CXC chemokines in LUAD, we used GO enrichment analysis and KEGG pathway enrichment analysis to examine the biological behavior of differentially expressed CXC chemokines. Interestingly, we found that these genes were mainly involved in biological processes, including cytokine-cytokine interaction, chemokine signaling pathway, and IL-17 signaling pathway. Previous studies also showed that chemokines play important regulatory roles in oncogenic processes, including tumor proliferation, apoptosis, angiogenesis, metastasis, chemoresistance, and immunomodulation. ${ }^{27-29}$ Additionally, Chen et al discovered that IL-17 could inhibit the

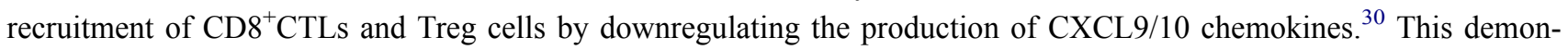
strates the feasibility of interfering with or inhibiting the production of specific CXC inflammatory cytokines for malignancy prevention and immunotherapy.

We then investigated the regulators responsible for CXC family member dysregulation. Our data indicated that GGCACAT-(CXCL2), -ATAGGAA-(CXCL3), -TCCCCAC-(CXCL10), and -TACTTGA-(CXCL17) could act as binding sites for miRNA to regulate transcription in gene fragments of CXC chemokine members. Next, we found that $\mathrm{CXC}$ chemokines family members were associated with kinase networks, including SRC family tyrosine kinases (LCK and FGR), serine/threonine protein kinases (ATM and PIM1), and mitogen-activated protein kinases (MAPK3). These kinases contribute to cell motility, ${ }^{31}$ differentiation, ${ }^{32}$ proliferation, and epithelial-mesenchymal transition. ${ }^{33}$ Furthermore, some of these kinases are essential regulators of cell migration and apoptosis and promote cancer metastasis. ${ }^{34}$ In NSCLC, these kinases have been observed to induce cell cycle arrest, modulate epithelial-mesenchymal transformation, and significantly alter tumor growth in lung cancer xenograft models, indicating that these kinases could promote tumor proliferation and metastasis. ${ }^{35}$

We also attempted to explore the key transcription regulators that mediate the differential expression of $\mathrm{CXC}$ chemokines and found that RELA, NFкB1, and SP1 might play a crucial role in the regulation of the gene expression of CXC chemokines. Persistent activation of RELA phosphorylation in lung tumorigenesis has been reported. ${ }^{36,37} \mathrm{Zhang}$ et al revealed a positive correlation between RELA and clinicopathological characteristics, including tumor grade, and metastasis system stage. And they reported that nuclear RELA led to poor prognosis in lung cancer patients through an NF- $\kappa$ B-positive feedback loop. ${ }^{38} \mathrm{NF}-\kappa \mathrm{B} 1$, a master regulator of inflammation, has been shown to play a key role in the growth, invasion, and metastasis of tumor cells. ${ }^{39}$ Garg et al highlighted the role of the NF- $\kappa \mathrm{B}$ pathway in inhibiting $\mathrm{T}$ cell infiltration and immune-mediated cancer cell death. They revealed that enhanced NF- $\kappa \mathrm{B} 1$ activity in pancreatic stromal cells promoted tumor cell growth by promoting the overexpression of CXCL12, which in turn prevented cytotoxic T cells from infiltrating and killing tumor cells. ${ }^{40} \mathrm{SP} 1$, a nuclear transcription factor, affects tumor angiogenesis (transforming growth factor- $\beta$ ) and metastasis (vascular endothelial growth factor) by regulating the expression of tumorrelated genes. ${ }^{41} \mathrm{SP} 1$ has also been found to be one of the cis-acting elements that regulate the transcription of CXCL1, ${ }^{42}$ and downregulation of the SP1 axis has been shown to inhibit the proliferation and metastasis of NSCLC through the AKT and ERK1/2 signaling pathways. ${ }^{43}$ Thus, our results might provide more guidance and evidence for the AKT $\rightarrow$ NF$\kappa \mathrm{B} / \mathrm{ERK} 1 / 2 \rightarrow \mathrm{CXCL}$ cascading signaling pathway in LUAD.

Many studies of the tumor microenvironment indicate that tumor-infiltrating immune cells play a crucial role in the progression and aggressiveness of tumors. ${ }^{44,45}$ In breast cancer ${ }^{46}$ and colorectal cancer, ${ }^{47}$ immunological parameters 
were shown to be better predictors of clinical outcomes than tumor-node-metastasis (TNM) staging. Hiraoka et al found that infiltration of $\mathrm{CD} 8^{+} \mathrm{T}$ cells and $\mathrm{CD} 4^{+} \mathrm{T}$ cells in NSCLC could synergistically inhibit tumor progression, and their cooccurrence was an independent prognostic factor favorable for lung cancer. ${ }^{48}$ Tumor-associated macrophages exert complex dual functions in their interactions with tumor cells, and Liu et al revealed that they may be involved in an important part of the inflammatory circuit in the tumor microenvironment, thus promoting tumor progression. ${ }^{49}$ Our study also found that differential expression of CXC chemokines was significantly correlated with the infiltration of 6 immune cell types, including B cells, CD8+T cells, CD4+T cells, macrophages, neutrophils, and dendritic cells. This suggests that the interaction between CXC chemokines and inflammatory cells is widely involved in the tumorigenesis inflammatory response pathway and that CXC chemokines could be used as a new immunological parameter to evaluate the prognosis of lung cancer patients.

To investigate the mechanism of CXCL17 abnormal expression in LUAD, PanglaoDB dataset was utilized to performed single-cell RNA sequencing localization analysis. We found that CXCL17 was enriched in pulmonary alveolar type II cell clusters. The reduction of CXCL17 expression in pulmonary alveolar type II cells played an important role in the carcinogenesis of LUAD. These results also provided new direction for our next research on the immune signaling pathway of CXCL17 in pulmonary alveolar type II cells and the down-regulation mechanism of CXCL17 in tumorigenicity.

There were also some limitations in our study. For instance, all the data in our study were obtained from different online databases, which inevitably caused background heterogeneity. Therefore, more studies with less heterogeneity and a larger sample size are needed to confirm our findings. Additionally, further independent-cohort and in vitro or in vivo studies should be conducted to validate our results.

\section{Conclusion}

In summary, our objective was to identify the aberrant expression profiles and prognostic biomarkers of the CXC chemokine family in patients with LUAD. We hope that the results of our study can help clinicians select more suitable and accurate prognostic biomarkers. Our detailed analysis of regulatory factor networks for CXC chemokine gene expression may provide novel insights into selecting potential immunotherapeutic targets. The mechanism of abnormal CXCL17 expression in pulmonary alveolar type II cells and its role in carcinogenesis is a new topic worth further investigating.

\section{Ethical Statement}

This study was approved in accordance with the Ethical standards of the Institutional Ethics Committee of Qilu Hospital of Shandong University.

\section{Acknowledgments}

We thank English language editors: J. Chapnick and J. Gray.

\section{Author Contributions}

All authors made a significant contribution to the work reported, whether that is in the conception, study design, execution, acquisition of data, analysis and interpretation, or in all these areas; took part in drafting, revising or critically reviewing the article; gave final approval of the version to be published; have agreed on the journal to which the article has been submitted; and agree to be accountable for all aspects of the work.

\section{Funding}

This work was supported by the Taishan Scholar Program of Shandong province (no.ts201712087), the Major Science and Technology Innovation Project of Shandong Province (no. 2020CXGC011303), and the Clinical Research Project of Minimally Invasive Esophagectomy Based on Enhanced Rehabilitation Surgical Technology (no. 2020SDUCRCA013). 


\section{Disclosure}

The authors have no conflicts of interest for this work to declare.

\section{References}

1. Bray F, Ferlay J, Soerjomataram I, Siegel RL, Torre LA, Jemal A. Erratum: Global cancer statistics. 2018: GLOBOCAN estimates of incidence and mortality worldwide for 36 cancers in 185 countries. CA Cancer J Clin. 2020;70(4):313.

2. Senosain MF, Massion PP. Intratumor heterogeneity in early lung adenocarcinoma. Front Oncol. 2020;10:349. doi:10.3389/fonc.2020.00349

3. Duma N, Santana-Davila R, Molina JR. Non-small cell lung cancer: epidemiology, screening, diagnosis, and treatment. Mayo Clinic Proc. 2019;94 (8):1623-1640. doi:10.1016/j.mayocp.2019.01.013

4. Balkwill F. Cancer and the chemokine network. Nat Rev Cancer. 2004;4(7):540-550. doi:10.1038/nrc1388

5. Vandercappellen J, Van Damme J, Struyf S. The role of CXC chemokines and their receptors in cancer. Cancer Lett. 2008;267(2):226-244. doi:10.1016/j.canlet.2008.04.050

6. Nagarsheth N, Wicha MS, Zou W. Chemokines in the cancer microenvironment and their relevance in cancer immunotherapy. Nat Rev Immunol. 2017;17(9):559-572. doi:10.1038/nri.2017.49

7. Rivas-Fuentes S, Salgado-Aguayo A, Pertuz Belloso S, Gorocica Rosete P, Alvarado-Vásquez N, Aquino-Jarquin G. Role of chemokines in non-small cell lung cancer: angiogenesis and inflammation. J Cancer. 2015;6(10):938-952. doi:10.7150/jca.12286

8. Spaks A, Svirina D, Spaka I, et al. CXC chemokine ligand 4 (CXCL4) is predictor of tumour angiogenic activity and prognostic biomarker in non-small cell lung cancer (NSCLC) patients undergoing surgical treatment. Biomarkers. 2016;21(5):474-478. doi:10.3109/ 1354750X.2016.1172111

9. Meyerson M, Gabriel S, Getz G. Advances in understanding cancer genomes through second-generation sequencing. Nat Rev Genet. 2010;11 (10):685-696. doi:10.1038/nrg2841

10. Li T, Fan J, Wang B, et al. TIMER: a web server for comprehensive analysis of tumor-infiltrating immune cells. Cancer Res. 2017;77(21):e108e110. doi:10.1158/0008-5472.CAN-17-0307

11. Chandrashekar DS, Bashel B, Balasubramanya SAH, et al. UALCAN: a portal for facilitating tumor subgroup gene expression and survival analyses. Neoplasia. 2017;19(8):649-658. doi:10.1016/j.neo.2017.05.002

12. Tang Z, Li C, Kang B, Gao G, Li C, Zhang Z. GEPIA: a web server for cancer and normal gene expression profiling and interactive analyses. Nucleic Acids Res. 2017;45(W1):W98-w102. doi:10.1093/nar/gkx247

13. Gao J, Aksoy BA, Dogrusoz U, et al. Integrative analysis of complex cancer genomics and clinical profiles using the cBioPortal. Sci Signal. 2013;6 (269):pl1. doi:10.1126/scisignal.2004088

14. Warde-Farley D, Donaldson SL, Comes O, et al. The GeneMANIA prediction server: biological network integration for gene prioritization and predicting gene function. Nucleic Acids Res. 2010;38(WebServer issue):W214-W220. doi:10.1093/nar/gkq537

15. Szklarczyk D, Gable AL, Lyon D, et al. STRING v11: protein-protein association networks with increased coverage, supporting functional discovery in genome-wide experimental datasets. Nucleic Acids Res. 2019;47(D1):D607-D613. doi:10.1093/nar/gky1131

16. Liao Y, Wang J, Jaehnig EJ, Shi Z, Zhang B. WebGestalt 2019: gene set analysis toolkit with revamped UIs and APIs. Nucleic Acids Res. 2019;47 (W1):W199-w205. doi:10.1093/nar/gkz401

17. Vasaikar SV, Straub P, Wang J, Zhang B. LinkedOmics: analyzing multi-omics data within and across 32 cancer types. Nucleic Acids Res. 2018;46 (D1):D956-d963. doi:10.1093/nar/gkx1090

18. Han H, Cho JW, Lee S, et al. TRRUST v2: an expanded reference database of human and mouse transcriptional regulatory interactions. Nucleic Acids Res. 2018;46(D1):D380-d386. doi:10.1093/nar/gkx1013

19. Franzén O, Gan LM, Björkegren JLM. PanglaoDB: a web server for exploration of mouse and human single-cell RNA sequencing data. Database. 2019;2019. doi:10.1093/database/baz046

20. Kuai Y, Liu H, Liu D, et al. An ultralow dose of the NADPH oxidase inhibitor diphenyleneiodonium (DPI) is an economical and effective therapeutic agent for the treatment of colitis-associated colorectal cancer. Theranostics. 2020;10(15):6743-6757. doi:10.7150/thno.43938

21. Vandercappellen J, Liekens S, Bronckaers A, et al. The COOH-terminal peptide of platelet factor-4 variant (CXCL4L1/PF-4var47-70) strongly inhibits angiogenesis and suppresses B16 melanoma growth in vivo. Mol Cancer Res. 2010;8(3):322-334. doi:10.1158/1541-7786.MCR-090176

22. Hu J, Zhao Q, Kong LY, et al. Regulation of tumor immune suppression and cancer cell survival by CXCL1/2 elevation in glioblastoma multiforme. Sci Adv. 2021;7(5). doi:10.1126/sciadv.abc2511

23. Yang M, Lu J, Zhang G, et al. CXCL13 shapes immunoactive tumor microenvironment and enhances the efficacy of PD-1 checkpoint blockade in high-grade serous ovarian cancer. J Immunother Cancer. 2021;9(1). doi:10.1136/jitc-2020-001136

24. Ikeda T, Nishita M, Hoshi K, Honda T, Kakeji Y, Minami Y. Mesenchymal stem cell-derived CXCL16 promotes progression of gastric cancer cells by STAT3-mediated expression of Ror1. Cancer Sci. 2020;111(4):1254-1265. doi:10.1111/cas.14339

25. Matsui A, Yokoo H, Negishi Y, et al. CXCL17 expression by tumor cells recruits CD11b+Gr1 high F4/80- cells and promotes tumor progression. PLoS One. 2012;7(8):e44080. doi:10.1371/journal.pone.0044080

26. Hu Y, Hu H, Miao L, et al. Multicenter study of diagnostic procedures, genetic aberration analysis, and first-line treatment of lung cancer in Jiangsu Province, China. Thoracic Cancer. 2018;9(3):376-383. doi:10.1111/1759-7714.12588

27. Roy I, Getschman AE, Volkman BF, Dwinell MB. Exploiting agonist biased signaling of chemokines to target cancer. Mol Carcinog. 2017;56 (3):804-813. doi:10.1002/mc.22571

28. Bian X, Xiao YT, Wu T, et al. Microvesicles and chemokines in tumor microenvironment: mediators of intercellular communications in tumor progression. Mol Cancer. 2019;18(1):50. doi:10.1186/s12943-019-0973-7

29. Bikfalvi A, Billottet C. The CC and CXC chemokines: major regulators of tumor progression and the tumor microenvironment. Am J Physiol Cell Physiol. 2020;318(3):C542-C554. doi:10.1152/ajpcell.00378.2019

30. Chen J, Ye X, Pitmon E, et al. IL-17 inhibits CXCL9/10-mediated recruitment of CD8(+) cytotoxic T cells and regulatory T cells to colorectal tumors. J Immunother Cancer. 2019;7(1):324. doi:10.1186/s40425-019-0757-z 
31. Rettig M, Trinidad K, Pezeshkpour G, et al. PAK1 kinase promotes cell motility and invasiveness through CRK-II serine phosphorylation in non-small cell lung cancer cells. PLoS One. 2012;7(7):e42012. doi:10.1371/journal.pone.0042012

32. Pandolfi A, Stanley RF, Yu Y, et al. PAK1 is a therapeutic target in acute myeloid leukemia and myelodysplastic syndrome. Blood. $2015 ; 126$ (9):1118-1127. doi:10.1182/blood-2014-12-618801

33. Kouhkan F, Mobarra N, Soufi-Zomorrod M, et al. MicroRNA-129-1 acts as tumour suppressor and induces cell cycle arrest of GBM cancer cells through targeting IGF2BP3 and MAPK1. J Med Genet. 2016;53(1):24-33. doi:10.1136/jmedgenet-2015-103225

34. Zhu L, Yang S, Wang J. miR-217 inhibits the migration and invasion of HeLa cells through modulating MAPK1. Int J Mol Med. 2019;44 (5):1824-1832. doi:10.3892/ijmm.2019.4328

35. Chung JH, Kim DH, Kim YS, et al. Upregulation of P21-activated kinase 1 (PAK1)/CREB axis in squamous non-small cell lung carcinoma. Cel Physiol Biochem. 2018;50(1):304-316. doi:10.1159/000494007

36. Meylan E, Dooley AL, Feldser DM, et al. Requirement for NF-kappaB signalling in a mouse model of lung adenocarcinoma. Nature. 2009;462 (7269):104-107. doi:10.1038/nature08462

37. Bassères DS, Ebbs A, Levantini E, Baldwin AS. Requirement of the NF-kappaB subunit p65/RelA for K-Ras-induced lung tumorigenesis. Cancer Res. 2010;70(9):3537-3546. doi:10.1158/0008-5472.CAN-09-4290

38. Zhang D, Jin X, Wang F, et al. Combined prognostic value of both RelA and IkappaB-alpha expression in human non-small cell lung cancer. Ann Surg Oncol. 2007;14(12):3581-3592. doi:10.1245/s10434-007-9560-Z

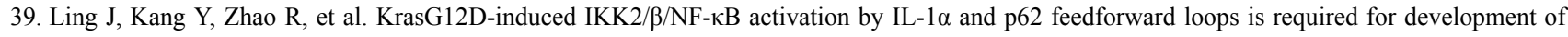
pancreatic ductal adenocarcinoma. Cancer Cell. 2012;21(1):105-120. doi:10.1016/j.ccr.2011.12.006

40. Garg B, Giri B, Modi S, et al. NFкB in Pancreatic Stellate Cells Reduces Infiltration of Tumors by Cytotoxic T Cells and Killing of Cancer Cells, via Up-regulation of CXCL12. Gastroenterology. 2018;155(3):880-891.e888. doi:10.1053/j.gastro.2018.05.051

41. Deng R, Mo F, Chang B, et al. Glucose-derived AGEs enhance human gastric cancer metastasis through RAGE/ERK/Sp1/MMP2 cascade. Oncotarget. 2017;8(61):104216-104226. doi:10.18632/oncotarget.22185

42. Curry JM, Eubank TD, Roberts RD, et al. M-CSF signals through the MAPK/ERK pathway via Sp1 to induce VEGF production and induces angiogenesis in vivo. PLoS One. 2008;3(10):e3405. doi:10.1371/journal.pone.0003405

43. Li X, Fu Y, Xia X, et al. Knockdown of SP1/Syncytin1 axis inhibits the proliferation and metastasis through the AKT and ERK1/2 signaling pathways in non-small cell lung cancer. Cancer Med. 2019;8(12):5750-5759. doi:10.1002/cam4.2448

44. Stankovic B, Bjørhovde HAK, Skarshaug R, et al. Immune Cell Composition in Human Non-small Cell Lung Cancer. Front Immunol. 2018 ;9:3101. doi:10.3389/fimmu.2018.03101

45. Barnes TA, Amir E. HYPE or HOPE: the prognostic value of infiltrating immune cells in cancer. Br J Cancer. 2018;118(2):e5. doi:10.1038/ bjc. 2017.417

46. Mahmoud SM, Paish EC, Powe DG, et al. Tumor-infiltrating CD8+ lymphocytes predict clinical outcome in breast cancer. J Clin Oncol. 2011;29 (15):1949-1955. doi:10.1200/JCO.2010.30.5037

47. Zhang L, Conejo-Garcia JR, Katsaros D, et al. Intratumoral T cells, recurrence, and survival in epithelial ovarian cancer. $N$ Engl J Med. 2003;348 (3):203-213. doi:10.1056/NEJMoa020177

48. Hiraoka K, Miyamoto M, Cho Y, et al. Concurrent infiltration by CD8+ T cells and CD4+ T cells is a favourable prognostic factor in non-small-cell lung carcinoma. Br J Cancer. 2006;94(2):275-280. doi:10.1038/sj.bjc.6602934

49. Liu Y, Cao X. The origin and function of tumor-associated macrophages. Cell Mol Immunol. 2015;12(1):1-4. doi:10.1038/cmi.2014.83

International Journal of General Medicine

\section{Publish your work in this journal}

The International Journal of General Medicine is an international, peer-reviewed open-access journal that focuses on general and internal medicine, pathogenesis, epidemiology, diagnosis, monitoring and treatment protocols. The journal is characterized by the rapid reporting of reviews, original research and clinical studies across all disease areas. The manuscript management system is completely online and includes a very quick and fair peer-review system, which is all easy to use. Visit http://www.dovepress.com/testimonials.php to read real quotes from published authors.

Submit your manuscript here: https://www.dovepress.com/international-journal-of-general-medicine-journal 